\title{
Pontryagin classes of locally symmetric manifolds
}

\author{
BENA TSHISHIKU
}

\begin{abstract}
Pontryagin classes $p_{i}(M)$ are basic invariants of a smooth manifold $M$, and many topological problems can be reduced to computing these classes. For a locally symmetric manifold, Borel and Hirzebruch gave an algorithm to determine if $p_{i}(M)$ is nonzero. In addition they implemented their algorithm for a few well-known $M$ and for $i=1,2$. Nevertheless, there remained several $M$ for which their algorithm was not implemented. In this note we compute low-degree Pontryagin classes for every closed, locally symmetric manifold of noncompact type. As a result of this computation, we answer the question: Which closed locally symmetric $M$ have at least one nonzero Pontryagin class?
\end{abstract}

57R20; 06B15

\section{Introduction}

For a manifold $M$ and $i>0$, the Pontryagin class $p_{i}(M) \in H^{4 i}(M ; \mathbb{Q})$ is a diffeomorphism invariant. When these classes are nonzero, they can serve as obstructions to certain geometric problems (see Tshishiku [18], for example).

In this paper, we are interested in closed locally symmetric manifolds $M$ of noncompact type. Let $G$ be a semisimple Lie group without compact factors; let $K \subset G$ be a maximal compact subgroup; and let $\Gamma \subset G$ be a cocompact, torsion-free lattice. The manifold $G / K$ has a $G$-invariant Riemannian metric and is a symmetric space of noncompact type. $\Gamma$ acts freely and properly on $G / K$, and the closed manifold $M=\Gamma \backslash G / K$ is a locally symmetric manifold of noncompact type.

Question 1.1 For which $\Gamma \backslash G / K$ is $p_{i}(\Gamma \backslash G / K) \neq 0$ for some $i>0$ ?

Throughout this paper all cohomology groups $H^{*}(\cdot)$ will be with $\mathbb{Q}$ coefficients unless otherwise specified.

The classical approach to determine if $p_{i}(\Gamma \backslash G / K) \neq 0$ is roughly as follows. Let $U \subset G_{\mathbb{C}}$ be the maximal compact subgroup of the complexification of $G$. By the proportionality principle (see Kamber and Tondeur [10, Section 4.14]), $p_{i}(\Gamma \backslash G / K) \neq 0$ if and only if $p_{i}(U / K) \neq 0$. Borel and Hirzebruch [4] relate $p_{i}(U / K)$ to the weights of 
the action of $K$ on the Lie algebra $\operatorname{Lie}(U)$. From this, showing $p_{i}(U / K) \neq 0$ reduces to showing that a polynomial is nonzero modulo an ideal; see [4] for details, which also contains explicit computations, including computations that $p_{1}\left(F_{4} / \operatorname{Spin}_{9}\right) \neq 0$, $p_{1}\left(G_{2} / \mathrm{SO}_{4}\right) \neq 0$, and that the first Chern class $c_{1}(U / K) \neq 0$, where $U / K$ is a compact Hermitian symmetric manifold and $U$ is not of exceptional type.

The objective of this paper is three-fold:

(1) Give a stream-lined way to determine if $p_{i}(\Gamma \backslash G / K) \neq 0$. One feature of our approach is that it does not use the proportionality principle mentioned above to reduce to computations for the compact dual $U / K$. Our main idea is to use the action of $\Gamma$ on the visual boundary $\partial(G / K)$ to study Pontryagin classes. This differs from the approach of Borel and Hirzebruch but ultimately reduces to the same problem: determining if a polynomial is nonzero modulo an ideal.

(2) Compute low-dimensional Pontryagin classes $p_{i}(\Gamma \backslash G / K)$ for every locally symmetric manifold of noncompact type, including every exceptional example. As mentioned above, for a handful of $G$ these computations follow from computations done by Borel and Hirzebruch [4]. Computations for $G=E_{6(6)}$ and $G=E_{6(-26)}$ were done by Takeuchi [17]; however, the author was unable to find computations for every semisimple Lie group. The purpose of the present paper is to have a single source for computations for every $\Gamma \backslash G / K$.

(3) Answer Question 1.1. A priori the answer depends on the choice of both $\Gamma$ and $G$, but it follows from the proportionality principle that the answer is independent of $\Gamma$ (here it is important that $\Gamma$ is cocompact). The author's interest in Question 1.1 arose from Tshishiku [18], in which Theorem 1.2 below is used to address a Nielsen realization problem: if $p_{i}(\Gamma \backslash G / K) \neq 0$ for some $i>0$, then the natural homomorphism $\pi_{1}(M) \rightarrow \pi_{0} \operatorname{Diff}(M, *)$ does not lift to $\operatorname{Diff}(M, *)$.

Main results A complete list of the simple real Lie groups of noncompact type are contained in Tables 1 and 2 below. We use subscripts instead of parentheses wherever possible; for example, we use $O_{n}$ instead of $O(n)$ to denote the orthogonal group. The examples in Table 1 are complex Lie groups, viewed here as real Lie groups. For any locally symmetric space $M$ of noncompact type, the universal cover $\tilde{M}$ is a symmetric space. Up to isogeny the isometry group of $\widetilde{M}$ has identity component equal to a product of groups in Tables 1 and 2. See Helgason [8] for further information.

For $G$ in Table 1 , we can conclude $p_{i}(\Gamma \backslash G / K)=0$ for all $i>0$ without any computation (see Section 5). This is why we have separated the simple, real Lie groups into two separate tables. The main work involved in the present paper is to determine those $G$ in Table 2 for which $p_{i}(\Gamma \backslash G / K)=0$ for all $i>0$. Here is a summary of our results: 


\begin{tabular}{cc}
\hline$G$ & $K$ \\
\hline $\mathrm{SL}_{n} \mathbb{C}$ & $\mathrm{SU}_{n}$ \\
$\mathrm{SO}_{n} \mathbb{C}$ & $\mathrm{SO}_{n}$ \\
$\mathrm{Sp}_{2 n} \mathbb{C}$ & $\mathrm{Sp}_{n}$ \\
$E_{6}^{\mathbb{C}}$ & $E_{6}$ \\
$E_{7}^{\mathbb{C}}$ & $E_{7}$ \\
$E_{8}^{\mathbb{C}}$ & $E_{8}$ \\
$F_{4}^{\mathbb{C}}$ & $F_{4}$ \\
$G_{2}^{\mathbb{C}}$ & $G_{2}$ \\
\hline
\end{tabular}

Table 1: Complex noncompact simple Lie groups

\begin{tabular}{ccc}
\hline & $G$ & $K$ \\
\hline AI & $\mathrm{SL}_{n} \mathbb{R}$ & $\mathrm{SO}_{n}$ \\
AII & $\mathrm{SU}_{2 n}^{*}$ & $\mathrm{Sp}_{n}$ \\
$\mathrm{AIII}$ & $\mathrm{SU}_{p, q}$ & $S\left(U_{p} \times U_{q}\right)$ \\
$\mathrm{BDI}$ & $\mathrm{SO}_{p, q}$ & $\mathrm{SO}_{p} \times \mathrm{SO}_{q}$ \\
$\mathrm{DIII}$ & $\mathrm{SO}_{2 n}^{*}$ & $U_{n}$ \\
$\mathrm{CI}$ & $\mathrm{Sp}_{2 n} \mathbb{R}$ & $U_{n}$ \\
$\mathrm{CII}$ & $\mathrm{Sp}_{p, q}$ & $\mathrm{Sp}_{p} \times \mathrm{Sp}_{q}$ \\
$\mathrm{E}_{6} \mathrm{I}$ & $E_{6(6)}$ & $\mathfrak{s p}_{4}$ \\
$\mathrm{E}_{6} \mathrm{II}$ & $E_{6(2)}$ & $\mathfrak{s u}_{6} \times \mathfrak{s u}_{2}$ \\
$\mathrm{E}_{6} \mathrm{III}$ & $E_{6(-14)}$ & $\mathfrak{s o}_{10} \times \mathfrak{s o}_{2}$ \\
$\mathrm{E}_{6} \mathrm{IV}$ & $E_{6(-26)}$ & $\mathfrak{f}_{4}$ \\
$\mathrm{E}_{7} \mathrm{~V}$ & $E_{7(7)}$ & $\mathfrak{s u}_{8}$ \\
$\mathrm{E}_{7} \mathrm{VI}$ & $E_{7(-5)}$ & $\mathfrak{s o}_{12} \times \mathfrak{s u}_{2}$ \\
$\mathrm{E}_{7} \mathrm{VII}$ & $E_{7(-25)}$ & $\mathfrak{e}_{6} \times \mathfrak{s o}_{2}$ \\
$\mathrm{E}_{8} \mathrm{VIII}$ & $E_{8(8)}$ & $\mathfrak{s o}_{16}$ \\
$\mathrm{E}_{8} \mathrm{IX}$ & $E_{8(-24)}$ & $\mathfrak{e}_{7} \times \mathfrak{s u}_{2}$ \\
$\mathrm{~F}_{4} \mathrm{I}$ & $F_{4(4)}$ & $\mathfrak{s p}_{3} \times \mathfrak{s u}_{2}$ \\
$\mathrm{~F}_{4} \mathrm{II}$ & $F_{4(-20)}$ & $\mathfrak{s o}_{9}$ \\
$\mathrm{G}_{2}$ & $G_{2(2)}$ & $\mathfrak{s u}_{2} \times \mathfrak{s u}_{2}$ \\
\hline & &
\end{tabular}

Table 2: Real noncompact simple Lie groups

Theorem 1.2 Let $G$ be any real, simple, noncompact Lie group and let $\Gamma \subset G$ be a cocompact lattice. Then $p_{i}(\Gamma \backslash G / K)=0$ for all $i>0$ if and only if $G$ is

(i) one of the Lie groups in Table 1, or

(ii) one of $\mathrm{SL}_{n}(\mathbb{R}), \mathrm{SU}_{2 n}^{*}, \mathrm{SO}_{p, 1}$ or $E_{6(-26)}$.

Theorem 1.2 and its proof (in Section 6) show that the answer to Question 1.1 is somewhat subtle. For example, let $G=\mathrm{SO}_{p, q}$ and assume $p \geq q$. If $p, q \geq 2$, then $p_{1}(\Gamma \backslash G / K) \neq 0$ if and only if $p \neq q$. If $p=q$, then $p_{2}(\Gamma \backslash G / K) \neq 0$ as long as $p \geq 4$. If either $p=q=2$ or $p=q=3$ or $p>q=1$, then $p_{i}(\Gamma \backslash G / K)=0$ for all $i>0$.

It turns out that if $G$ is not one of the groups from (i) or (ii) in Theorem 1.2, and $\Gamma \subset G$ is cocompact, then either $p_{1}(\Gamma \backslash G / K) \neq 0$ or $p_{2}(\Gamma \backslash G / K) \neq 0$. Thus to answer Question 1.1, we need only consider low-dimensional Pontryagin classes. To 
determine if $p_{i}(\Gamma \backslash G / K) \neq 0$ for $i \geq 3$ using the methods of this paper would be feasible, but more computationally intensive.

The proof of Theorem 1.2 immediately extends to the case when $G$ is semisimple to give a complete classification of which $\Gamma \backslash G / K$ have $p_{i}(\Gamma \backslash G / K)=0$ for all $i>0$.

Corollary 1.3 Let $G=\prod G_{i}$ be a semisimple Lie group with simple, noncompact factors $G_{i}$, and let $\Gamma \subset G$ be a cocompact lattice. Then $p_{i}(\Gamma \backslash G / K)=0$ for all $i>0$ if and only if each $G_{i}$ is one of the groups from (i) or (ii) in Theorem 1.2.

Instead of asking for $\Gamma \backslash G / K$ with a nonzero Pontryagin class, one could ask for $\Gamma \backslash G / K$ with a nonzero Pontryagin number. Specifically, let $M$ be a manifold with $\operatorname{dim} M=4 k$, and let $i_{1}, \ldots, i_{m} \in \mathbb{N}$ be such that $i_{1}+\cdots+i_{m}=k$. The cup product $p_{i_{1}}(M) \cup \cdots \cup p_{i_{m}}(M) \in H^{4 k}(M)$ can be evaluated on the fundamental class $[M] \in H_{4 k}(M)$, and the resulting integer

$$
\left\langle p_{i_{1}}(M) \cup \cdots \cup p_{i_{m}}(M),[M]\right\rangle \in \mathbb{Z}
$$

is called a Pontryagin number. These integers are topological invariants of $M$ by Novikov's theorem (see Lafont and Roy [12], for example). We remark that a manifold can have zero Pontryagin numbers but have some nonzero Pontryagin classes. For example, take $\Gamma \subset G=\mathrm{SO}_{p, q}$ with $p, q$ both odd. As explained in [12, Theorem B], the Pontryagin numbers of $\Gamma \backslash G / K$ are all zero. On the other hand, we show that if $p>q>2$, then $p_{1}(\Gamma \backslash G / K) \neq 0$ (see Section 6). For more information about Pontryagin numbers of locally symmetric spaces, see [12]. For recent results about the Euler characteristic of homogeneous spaces, see Mostow [16].

Method of proof We compute $p_{i}(\Gamma \backslash G / K)$ by the following procedure. Write $n=\operatorname{dim} M$. The unit tangent bundle $T^{1} M \rightarrow M$ has a flat $\operatorname{Homeo}\left(S^{n-1}\right)$ structure with monodromy $\mu: \Gamma \rightarrow \operatorname{Homeo}\left(S^{n-1}\right)$ given by the action of $\Gamma$ on the visual boundary $\partial(G / K) \simeq S^{n-1}$. The homomorphism $\mu$ induces a map of classifying spaces $M \approx B \Gamma \rightarrow B \operatorname{Homeo}\left(S^{n-1}\right)$ and hence a map

$$
\mu^{*}: H^{*}\left(B \operatorname{Homeo}\left(S^{n-1}\right)\right) \rightarrow H^{*}(M) \text {. }
$$

There are classes $q_{i} \in H^{4 i}\left(B \operatorname{Homeo}\left(S^{n-1}\right)\right)$ for which $\mu^{*}\left(q_{i}\right)=p_{i}(M)$; see Section 2 . To determine if $\mu^{*}\left(q_{i}\right)=0$, note that $\mu$ factors $\mu=\alpha_{1} \circ \alpha_{2} \circ \alpha_{3}$, where

$$
\Gamma \stackrel{\alpha_{3}}{\longrightarrow} G^{\delta} \stackrel{\alpha_{2}}{\longrightarrow} G \stackrel{\alpha_{1}}{\longrightarrow} \operatorname{Homeo}\left(S^{n-1}\right) .
$$

Here $G^{\delta}$ is the group $G$ viewed as a Lie group with the discrete topology. The map $\alpha_{3}$ is the inclusion, $\alpha_{2}$ is the identity (which is continuous), and $\alpha_{1}$ is the action of $G$ on its visual boundary. 
To understand $\mu^{*}=\left(\alpha_{1} \circ \alpha_{2} \circ \alpha_{3}\right)^{*}$, we study the individual maps

$$
H^{*}\left(B \text { Homeo }\left(S^{n-1}\right)\right) \stackrel{\alpha_{1}^{*}}{\longrightarrow} H^{*}(B G) \stackrel{\alpha_{2}^{*}}{\longrightarrow} H^{*}\left(B G^{\delta}\right) \stackrel{\alpha_{3}^{*}}{\longrightarrow} H^{*}(M) .
$$

Step 1 Computing $\alpha_{1}^{*}$ reduces to computing weights of the adjoint action of $K$ on $\mathfrak{g}=\operatorname{Lie}(G)$ (see Section 4).

Step 2 Computing $\alpha_{2}^{*}$ reduces to computing the map $H^{*}\left(B G_{\mathbb{C}}\right) \rightarrow H^{*}(B G)$ induced by complexification $G \rightarrow G_{\mathbb{C}}$. This follows from Chern-Weil theory (see Section 5).

Step $3 \alpha_{3}^{*}$ is injective on the image of $H^{*}(B G) \rightarrow H^{*}\left(B G^{\delta}\right)$ by a transfer argument (see Section 6).

Structure of the paper In Section 2 we define the classes $q_{i}$ mentioned above and show that $\mu^{*}\left(q_{i}\right)=p_{i}(M)$. In Section 3 we recall Borel's computation of $H^{*}(B K)$ for $K$ a compact Lie group, and we recall how characteristic classes of a representation can be computed in terms of the weights of that representation. In Sections 4 and 5 we complete Steps 1 and 2, respectively. In Section 6 we explain Step 3 and combine Steps 1,2 and 3 to conclude which $\Gamma \backslash G / K$ have $p_{i}(\Gamma \backslash G / K) \neq 0$ for some $i>0$ and thus prove Theorem 1.2.

Acknowledgement The author would like to thank his advisor B Farb for his gracious and ceaseless support, for his encouragement to complete this project and for extensive comments that significantly improved a draft of this paper. The author also thanks the referee for carefully reading this paper and pointing out several typographical errors.

\section{An algorithm for computing Pontryagin classes}

This section has two goals. First we recall the definition of the Pontryagin classes of topological sphere bundles $q_{i} \in H^{4 i}\left(B \operatorname{Homeo}\left(S^{n-1}\right)\right)$. Then we explain why $\mu^{*}\left(q_{i}\right)=p_{i}(M)$. This will follow from the construction of a flat $\operatorname{Homeo}\left(S^{n-1}\right)$ structure on the unit tangent bundle $T^{1} M \rightarrow M$.

\subsection{Pontryagin classes of sphere bundles}

The Pontryagin classes $p_{i} \in H^{*}\left(B O_{n}\right)$ are classically defined as invariants of real vector bundles (see Milnor and Stasheff [15]). The following proposition shows that these invariants can also be defined for topological $\mathbb{R}^{n}$-bundles.

Proposition 2.1 The inclusion $g$ : $O_{n} \hookrightarrow \operatorname{Homeo}\left(\mathbb{R}^{n}\right)$ induces a surjection

$$
g^{*}: H^{*}\left(B \operatorname{Homeo}\left(\mathbb{R}^{n}\right) ; \mathbb{Q}\right) \rightarrow H^{*}\left(B O_{n} ; \mathbb{Q}\right) .
$$


Proposition 2.1 can be proved using results from Kirby and Siebenmann [11]. The argument (which the author learned from A Hatcher) is given in [18].

From Proposition 2.1, Pontryagin classes of sphere bundles can be defined as follows. Define a homomorphism $\delta$ : $\operatorname{Homeo}\left(S^{n-1}\right) \rightarrow \operatorname{Homeo}\left(\mathbb{R}^{n}\right)$ using the Alexander trick: $\delta(f)$ performs the homeomorphism $f$ on the sphere of radius $r$ for every $r>0$, and $\delta(f)$ fixes the origin. This induces maps between classifying spaces and hence a map

$$
\delta^{*}: H^{*}\left(B \operatorname{Homeo}\left(\mathbb{R}^{n}\right)\right) \rightarrow H^{*}\left(B \operatorname{Homeo}\left(S^{n-1}\right)\right) .
$$

The restriction of $\delta$ to the subgroup $O_{n} \subset \operatorname{Homeo}\left(S^{n-1}\right)$ is the standard action $O_{n} \rightarrow \operatorname{Homeo}\left(\mathbb{R}^{n}\right)$, so there is a commutative diagram:

$$
H^{*}\left(B \text { Homeo }(\underbrace{\left.\left.\mathbb{R}^{n}\right)\right) \stackrel{\delta^{*}}{\longrightarrow} H^{*}\left(B O_{n}\right)}_{g^{*}} H^{r^{*}}\right.
$$

By Proposition 2.1, there is a class $\tilde{p}_{i} \in H^{4 i}\left(B \operatorname{Homeo}\left(\mathbb{R}^{n}\right)\right)$ with $g^{*}\left(\tilde{p}_{i}\right)=p_{i}$. Since diagram (3) commutes, $\delta^{*}\left(\tilde{p}_{i}\right) \in H^{4 i}\left(B \operatorname{Homeo}\left(S^{n-1}\right)\right)$ is nontrivial. We refer to the classes $q_{i}=\delta^{*}\left(\tilde{p}_{i}\right)$ as the Pontryagin classes of topological sphere bundles.

\subsection{Flat structure on the unit tangent bundle}

We continue to assume that $G$ is a semisimple Lie group without compact factors and that $K \subset G$ is a maximal compact subgroup. With these assumptions $G / K$ is contractible and has a metric of nonpositive curvature such that $G$ acts by isometries on $G / K$. In addition, $G$ acts on the visual boundary $\partial(G / K) \simeq S^{n-1}$ (see eg Ballman, Gromov and Schroeder [2]). If $G$ has rank 1 , then the action of $G$ on $\partial(G / K)$ is smooth, but this is not known in general. Thus, even though $G / K$ is an algebraic example of a contractible, nonpositively curved manifold, the action on $\partial(G / K)$ is a priori only an action by homeomorphisms. By restriction to $\Gamma \subset G$, we obtain an action $\Gamma \rightarrow \operatorname{Homeo}\left(S^{n-1}\right)$, and from this action we can build an $S^{n-1}$-bundle $E \rightarrow M$, where $E$ is the quotient of $(G / K) \times S^{n-1}$ by the diagonal action of $\Gamma$.

Lemma 2.2 Let $M$ be a complete Riemannian manifold of nonpositive curvature with universal cover $\tilde{M}$. The sphere bundle whose monodromy is the action of the deck group $\pi_{1}(M)$ on the visual boundary $\partial \tilde{M} \simeq S^{n-1}$ is isomorphic to the unit tangent bundle $T^{1} M \rightarrow M$.

Lemma 2.2 shows that $T^{1} M \rightarrow M$ is flat. It also explains why the monodromy $\mu: \Gamma \rightarrow \operatorname{Homeo}\left(S^{n-1}\right)$ factors as claimed in $(1): \mu$ is the $\Gamma$-action on $\partial(G / K)$, which is the restriction of the $G$-action on $\partial(G / K)$. 
Proof of Lemma 2.2 Since $M$ has nonpositive curvature, $\tilde{M}$ is contractible, and the unit tangent bundle $\pi: T^{1} \tilde{M} \rightarrow \tilde{M}$ is trivial. An explicit trivialization can be defined as follows. Define a map

$$
\phi: T^{1} \tilde{M} \rightarrow \partial \tilde{M}
$$

by associating a geodesic ray to a vector via the exponential map. Now define

$$
\begin{aligned}
\tau: T^{1} \tilde{M} & \rightarrow \tilde{M} \times \partial \tilde{M}, \\
z & \mapsto(\pi(z), \phi(z)) .
\end{aligned}
$$

Since $\partial \tilde{M}$ is homeomorphic to $S^{n-1}$, this gives the desired trivialization.

It is not hard to see that $\tau$ is equivariant with respect to the actions of the deck group $\pi_{1}(M)$ on $T^{1} \tilde{M}$ and $\tilde{M} \times \partial \tilde{M}$. Note that $T^{1} \tilde{M} / \pi_{1}(M) \simeq T^{1} M$. Then the quotient by the $\pi_{1}(M)$-action induces a bundle isomorphism:

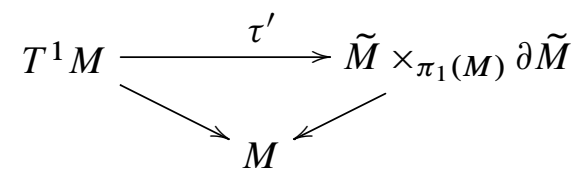

\section{Compact Lie groups, characteristic classes, and representations}

Let $K$ be a compact Lie group and let $B K$ be its classifying space. In this section we recall Borel's computation of the cohomology $H^{*}(B K)$ (see Theorem 3.1). For a representation $\rho: K \rightarrow \mathrm{GL}_{m}(\mathbb{C})$, we recall how to use Borel's computation to determine the image of $\rho^{*}: H^{*}\left(B \mathrm{GL}_{m}(\mathbb{C})\right) \rightarrow H^{*}(B K)$ (specifically the image of the Chern classes) in terms of the weights of $\rho$. For more details, see [4].

\subsection{The cohomology of $B K$}

Let $K$ be a compact Lie group. Let $S \subset K$ be a maximal abelian subgroup. $S$ is homeomorphic to an $r$-torus $\left(S^{1}\right)^{r}$ for some integer $r$, which is called the rank of $K$. Let $N_{K}(S)$ denote the normalizer of $S$ in $K$. The Weyl group is defined as $W=N_{K}(S) / S$.

Theorem 3.1 (Borel [3]) Let $K$ be a compact Lie group with maximal torus $S$ and Weyl group $W$. The inclusion $S \hookrightarrow K$ induces an isomorphism

$$
H^{*}(B K ; \mathbb{Q}) \simeq H^{*}(B S ; \mathbb{Q})^{W} .
$$




\subsection{Weights, transgression and characteristic classes}

Let $K$ be a compact Lie group with maximal torus $S$, and let $\rho: K \rightarrow \mathrm{GL}_{n}(\mathbb{C})$ be a representation. The restriction $\left.\rho\right|_{S}$ is a sum of 1-dimensional representations $\lambda_{i}: S \rightarrow \mathbb{C}^{\times}$, called the weights of $\rho$. By the identification $\mathbb{C}^{\times}=K(\mathbb{Z}, 1)$ and the fact that cohomology is a represented functor, the weights can be viewed as elements of $H^{1}(S ; \mathbb{Z})$. Since we are interested in cohomology with $\mathbb{Q}$-coefficients, we view the weights as elements of $H^{1}(S)=H^{1}(S ; \mathbb{Q})$.

For the representation $\rho$, we are interested in computing the induced map

$$
\rho^{*}: H^{*}\left(B \mathrm{GL}_{n}(\mathbb{C}) ; \mathbb{Q}\right) \rightarrow H^{*}(B K ; \mathbb{Q})
$$

in terms of the weights $\lambda_{1}, \ldots, \lambda_{n} \in H^{1}(S ; \mathbb{Q})$. Let $S \rightarrow E S \rightarrow B S$ be the universal principal $S$-bundle. The edge map

$$
\tau: H^{1}(S ; \mathbb{Q}) \rightarrow H^{2}(B S ; \mathbb{Q})
$$

on the $E_{2}$-page of the Serre spectral sequence of this fibration is an isomorphism, called the transgression. Let $w_{i}=-\tau\left(\lambda_{i}\right)$. The total Chern class $c(\rho) \in H^{*}(B S ; \mathbb{Q})$ is defined by

$$
c(\rho)=1+c_{1}(\rho)+\cdots+c_{n}(\rho)=\prod_{i=1}^{n}\left(1+w_{i}\right) .
$$

The Weyl group $W$ permutes the weights of $\rho$, so $c(\rho) \in H^{*}(B S)^{W} \simeq H^{*}(B K)$.

If $\rho: K \rightarrow \mathrm{GL}_{n}(\mathbb{R})$ is a real representation and $\rho_{\mathbb{C}}: K \rightarrow \mathrm{GL}_{n}(\mathbb{C})$ is the complexification, one defines the $i^{\text {th }}$ Pontryagin class $p_{i}(\rho)$ of $\rho$ by the formula

$$
p_{i}(\rho)=(-1)^{i} c_{2 i}\left(\rho_{\mathbb{C}}\right)
$$

Remark The transgression $\tau$ can be given concretely as follows. Make the identification $H^{1}(H ; \mathbb{Z}) \simeq \operatorname{Hom}\left(H, \mathbb{C}^{\times}\right)$, and define $\operatorname{Hom}\left(S, \mathbb{C}^{\times}\right) \rightarrow H^{2}(B S)$ : Given $\varphi: S \rightarrow \mathbb{C}^{\times}$, form the space

$$
E_{\varphi}=\frac{E H \times \mathbb{C}^{\times}}{H},
$$

which is the quotient of $E H \times \mathbb{C}^{\times}$by the diagonal action of $H$, where $H$ acts on $\mathbb{C}^{\times}$ by $\varphi$. Now $E_{\varphi}$ has a natural projection to $E H / H=B H$, and this makes $E_{\varphi}$ a $\mathbb{C}^{\times}$-bundle over $B H$. The first Chern class $c_{1}\left(E_{\varphi}\right)$ lives in $H^{2}(B S ; \mathbb{Z})$, and the transgression is given by $\tau(\varphi)=c_{1}\left(E_{\varphi}\right)$. 


\subsection{The invariant polynomials $H^{*}(B S)^{W}$}

Let $K$ be a compact Lie group with maximal torus $S$. The ring of invariant polynomials $H^{*}(B S)^{W}$ is well known (see Humphreys [9, Chapter 3]). For the exceptional groups, explicit polynomial generators for $H^{*}(B S)^{W}$ can be computed as follows. Let $K$ be one of the exceptional compact Lie groups: $E_{8}, E_{7}, E_{6}, F_{4}, G_{2}$. Let $V$ be a fundamental representation of $K$ of minimal dimension. Denote by $\lambda_{1}, \ldots, \lambda_{d} \in H^{1}(S)$ the weights of this representation, so that $\tau\left(\lambda_{i}\right) \in H^{2}(B S)$. Mehta [13] shows that power sums

$$
I_{k}=\sum_{i=1}^{d} \tau\left(\lambda_{i}\right)^{k}
$$

generate the invariant polynomials $H^{*}(B S)^{W}$. In the remainder of this section we recall the descriptions of $H^{*}(B S)^{W}$ for the different compact Lie groups, and we record explicit generators of $H^{*}(B S)^{W}$ that will be used in Sections 5 and 6.

To express the weights $\lambda_{1}, \ldots, \lambda_{d}$ for the exceptional $K$, we use the descriptions from Adams [1]. We remark that our expressions for $I_{k}$ for $E_{8}, E_{7}, E_{6}$ and $F_{4}$ agree with [13] up to a change of basis. Let $\operatorname{Sym}\left(x_{1}, \ldots, x_{n}\right) \subset \mathbb{Q}\left[x_{1}, \ldots, x_{n}\right]$ denote the subring of symmetric polynomials.

$\boldsymbol{B S O}_{\boldsymbol{n}}$ Let $k=\lfloor n / 2\rfloor$. As a subring of $\mathbb{Q}\left[y_{1}, \ldots, y_{k}\right]$,

$$
H^{*}\left(B \mathrm{SO}_{n}\right) \simeq H^{*}(B S)^{W}= \begin{cases}\operatorname{Sym}\left(y_{1}^{2}, \ldots, y_{k}^{2}\right) & \text { if } n=2 k+1, \\ \left\langle\operatorname{Sym}\left(y_{1}^{2}, \ldots, y_{k}^{2}\right), y_{1} \cdots y_{k}\right\rangle & \text { if } n=2 k .\end{cases}
$$

$\boldsymbol{B} \boldsymbol{U}_{\boldsymbol{n}}$

$$
H^{*}\left(B U_{n}\right) \simeq H^{*}(B S)^{W}=\operatorname{Sym}\left(y_{1}, \ldots, y_{n}\right) .
$$

Note that $H^{*}\left(B \mathrm{SU}_{n}\right)$ is the quotient of $\operatorname{Sym}\left(y_{1}, \ldots, y_{n}\right)$ by the ideal generated by $y_{1}+\cdots+y_{n}$. Similarly, $H^{*}\left(B S\left(U_{p} \times U_{q}\right)\right)$ is the quotient of

$$
\operatorname{Sym}\left(y_{1}, \ldots, y_{p}\right) \otimes \operatorname{Sym}\left(z_{1}, \ldots, z_{q}\right)
$$

by the ideal generated by $y_{1}+\cdots+y_{p}+z_{1}+\cdots+z_{q}$.

$$
\boldsymbol{B S P}_{\boldsymbol{n}} \quad H^{*}(B \operatorname{Sp}(n)) \simeq H^{*}(B S)^{W}=\operatorname{Sym}\left(y_{1}^{2}, \ldots, y_{n}^{2}\right) .
$$

$\boldsymbol{B E}_{\mathbf{8}}$ Let $S \subset E_{8}$ be a maximal torus with Weyl group $W$. Up to conjugation, we can assume $S \subset \operatorname{Spin}_{16} \subset E_{8}$ and that $S$ is a maximal torus of $\operatorname{Spin}_{16}$. This allows us to identify $H^{1}(S) \simeq \mathbb{Q}\left\{J_{1}, \ldots, J_{8}\right\}$ and $H^{2}(B S) \simeq \mathbb{Q}\left[z_{1}, \ldots, z_{8}\right]$ in such a way that the roots of $E_{8}$ are

$$
\begin{cases} \pm J_{i} \pm J_{j} & \text { if } 1 \leq i<j \leq 8 \\ \frac{1}{2}\left( \pm J_{1} \pm \cdots \pm J_{8}\right) & \text { if there are an even number of negations. }\end{cases}
$$


See [1, page 56]. $W$ preserves the roots, and $H^{*}(B S)^{W} \simeq H^{*}\left(B E_{8}\right)$ is generated by

$$
I_{2 k}=\sum_{1 \leq i<j \leq 8}\left(\left(z_{i}+z_{j}\right)^{2 k}+\left(z_{i}-z_{j}\right)^{2 k}\right)+\sum \frac{1}{2^{2 k}}\left(z_{1} \pm \cdots \pm z_{8}\right)^{2 k},
$$

where the second sum is over all terms with an even number of negations. According to [13, page 1088], $H^{*}(B S)^{W} \simeq \mathbb{Q}\left[I_{2}, I_{8}, I_{12}, I_{14}, I_{18}, I_{20}, I_{24}, I_{30}\right]$. We record here that $I_{2}=30\left(z_{1}^{2}+\cdots+z_{8}^{2}\right)$.

$\boldsymbol{B E _ { 7 }}$ For any embedding $\mathrm{SU}_{2} \hookrightarrow E_{8}$, the identity component of the centralizer of $\mathrm{SU}_{2}$ is isomorphic to $E_{7}$ (see [1, page 49]). Choose $\mathrm{SU}_{2}$ so that its roots are $\pm\left(J_{7}-J_{8}\right)$ in $E_{8}$ (cf (5)). Let $S \subset E_{7}$ be a maximal torus with Weyl group $W$. Since the roots of $E_{7}$ are orthogonal to the roots of $\mathrm{SU}_{2}$, we can identify $H^{1}(S) \simeq \mathbb{Q}\left\{J_{1}, \ldots, J_{6}, J_{7}+\right.$ $\left.J_{8}\right\}$. Let $z_{i}=\tau\left(J_{i}\right)$ in $H^{2}(B S)$ for $1 \leq i \leq 6$ and let $z_{7}=\tau\left(J_{7}+J_{8}\right)$.

Following [1, page 52], the fundamental representation of $E_{7}$ is 56-dimensional, and by restricting this representation to $\mathfrak{s o}_{12} \times \mathfrak{s u}_{2} \subset \mathfrak{e}_{7}$, one can compute the weights:

$$
\begin{cases} \pm J_{i} \pm \frac{1}{2}\left(J_{7}+J_{8}\right) & \text { if } 1 \leq i \leq 6 \\ \frac{1}{2}\left( \pm J_{1} \pm \cdots \pm J_{6}\right) & \text { if an odd number of the } J_{i} \text { are negated. }\end{cases}
$$

$W$ preserves these weights, and $H^{*}(B S)^{W} \simeq H^{*}\left(B E_{7}\right)$ is generated by

$$
I_{2 k}=\sum_{i=1}^{6}\left(\frac{1}{2} z_{7}+z_{i}\right)^{2 k}+\left(\frac{1}{2} z_{7}-z_{i}\right)^{2 k}+\frac{1}{2^{2 k}} \sum\left(z_{1} \pm \cdots \pm z_{6}\right)^{2 k},
$$

where the second sum is over all terms with an odd number of minus signs. According to [13, page 1086], $H^{*}(B S)^{W} \simeq \mathbb{Q}\left[I_{2}, I_{6}, I_{8}, I_{10}, I_{12}, I_{14}, I_{18}\right]$. Note that for this description of $E_{7}$,

$$
I_{2}=6\left(z_{1}^{2}+\cdots+z_{6}^{2}\right)+3 z_{7}^{2} .
$$

A second computation for $\boldsymbol{B E}_{\mathbf{7}}$ We will also need the following description of $H^{*}\left(B E_{7}\right)$. This time choose $\mathrm{SU}_{2} \hookrightarrow E_{8}$ so that its roots are $\pm\left(J_{1}+\cdots+J_{8}\right)$. Let $S \subset E_{7}$ be a maximal torus with Weyl group $W . H^{1}(S)$ is the orthogonal complement of $J_{1}+\cdots+J_{8}$ in $\mathbb{Q}\left\{J_{1}, \ldots, J_{8}\right\}$. One can compute the weights (with respect to this description of $\left.H^{1}(S)\right)$ of the fundamental representation of $E_{7}$ by restricting to $\mathrm{SU}_{8} \subset E_{7}$ (see [1, page 69]). The weights are

$$
\pm\left(J_{i}+J_{j}-\frac{1}{4}\left(J_{1}+\cdots+J_{8}\right)\right), \quad 1 \leq i<j \leq 8 .
$$

Let $z_{i}=\tau\left(J_{i}\right) \in H^{2}(B S)$ for $1 \leq i \leq 8$. (Technically $J_{i}$ does not live in $H^{1}(S)$, so we really mean the restriction of the weight $J_{i}$ for $E_{8}$ to a weight for $E_{7}$.) 
$H^{*}(B S)^{W} \simeq H^{*}\left(B E_{7}\right)$ is generated by

$$
I_{2 k}=\sum_{1 \leq i<j \leq 8}\left(\left(z_{i}+z_{j}\right)-\frac{1}{4}\left(z_{1}+\cdots+z_{8}\right)\right)^{2 k} .
$$

As above, $H^{*}(B S)^{W}=\mathbb{Q}\left[I_{2}, I_{6}, I_{8}, I_{10}, I_{12}, I_{14}, I_{18}\right]$, and one computes

$$
I_{2}=\frac{3}{4}\left[7 \sum_{1 \leq i \leq 8} z_{i}^{2}+2 \sum_{1 \leq i<j \leq 8} z_{i} z_{j}\right] \text {. }
$$

$\boldsymbol{B E}_{6}$ For any embedding $\mathrm{SU}_{3} \hookrightarrow E_{8}$, the identity component of the centralizer of $\mathrm{SU}_{3}$ is isomorphic to $E_{6}$ (see [1, page 49]). Choose $\mathrm{SU}_{3}$ so that its roots are $\pm\left(J_{6}-J_{7}\right), \pm\left(J_{6}-J_{8}\right)$, and $\pm\left(J_{7}-J_{8}\right)$ (cf (5)). Let $S \subset E_{6}$ be a maximal torus with Weyl group $W$. Since the roots of $E_{6}$ are orthogonal to the roots of $\mathrm{SU}_{3}$, we can identify

$$
H^{1}(S) \simeq \mathbb{Q}\left\{J_{1}, \ldots, J_{5}, J_{6}+J_{7}+J_{8}\right\} .
$$

Let $z_{i}=\tau\left(J_{i}\right) \in H^{2}(B S)$ for $1 \leq i \leq 5$, and let $z_{6}=\tau\left(J_{6}+J_{7}+J_{8}\right)$.

$\operatorname{Lie}\left(E_{6}\right)=\mathfrak{e}_{6}$ has two 27-dimensional fundamental representations $U_{1}, U_{2}$. One can compute the weights of $U_{i}$ by restricting to $\mathfrak{s o}_{10} \times \mathfrak{s o}_{2} \subset \mathfrak{e}_{6}$. Following [1, page 53], $U_{1}=\xi^{-4}+\lambda_{10}^{1} \otimes \xi^{2}+\Delta^{+} \otimes \xi^{-1}$ and its weights are

$$
\begin{cases} \pm J_{i}+\frac{1}{3}\left(J_{6}+J_{7}+J_{8}\right) & \text { if } 1 \leq i \leq 5 \\ \frac{1}{2}\left( \pm J_{1} \pm \cdots \pm J_{5}-\frac{1}{3}\left(J_{6}+J_{7}+J_{8}\right)\right) & \text { if an even number of the } J_{i} \text { are negated, } \\ -\frac{2}{3}\left(J_{6}+J_{7}+J_{8}\right) . & \end{cases}
$$

$W$ preserves these weights, and $H^{*}(B S)^{W} \simeq H^{*}\left(B E_{6}\right)$ is generated by

(6) $I_{k}=\left(-\frac{2}{3} z_{6}\right)^{k}+\sum_{i=1}^{5}\left(\frac{1}{3} z_{6}+z_{i}\right)^{k}+\left(\frac{1}{3} z_{6}-z_{i}\right)^{k}+\frac{1}{2^{k}} \sum\left( \pm z_{1} \pm \cdots \pm z_{5}-\frac{1}{3} z_{6}\right)^{k}$.

In particular, $I_{1}=I_{3}=0, I_{2}=6\left(z_{1}^{2}+z_{2}^{2}+z_{3}^{2}+z_{4}^{2}+z_{5}^{2}\right)+2 z_{6}^{2}$ and $I_{4}=\frac{1}{12}\left(I_{2}\right)^{2}$. According to [13, page 1086], $H^{*}(B S)^{W} \simeq \mathbb{Q}\left[I_{2}, I_{5}, I_{6}, I_{8}, I_{9}, I_{12}\right]$.

A second computation for $\boldsymbol{B E}_{\mathbf{6}}$ We will also need the following description of $H^{*}\left(B E_{6}\right)$. This time choose $\mathrm{SU}_{3} \hookrightarrow E_{8}$ so that the roots are

$$
\left\{ \pm \frac{1}{2}\left(J_{1}+\cdots+J_{6}\right) \pm \frac{1}{2}\left(J_{7}+J_{8}\right), \pm\left(J_{7}+J_{8}\right)\right\} .
$$

Let $S \subset E_{6}$ be a maximal torus with Weyl group $W \cdot H^{1}(S)$ is the subspace of $\mathbb{Q}\left\{J_{1}, \ldots, J_{8}\right\}$ that is orthogonal to the roots in (7). 
For this description of $\mathfrak{e}_{6}$, we compute the weights of the fundamental representations $U_{1}$ and $U_{2}$ by restricting to $\mathfrak{s u}_{6} \times \mathfrak{s u}_{2} \subset \mathfrak{e}_{6}$. As an $\mathfrak{s u}_{6} \times \mathfrak{s u}_{2}$-representation,

$$
U_{1}=\lambda_{6}^{2}+\lambda_{6}^{5} \otimes \lambda_{2}^{1} \quad \text { and } \quad U_{2}=\lambda_{6}^{4}+\lambda_{6}^{1} \otimes \lambda_{2}^{1},
$$

where $\lambda_{m}^{i}$ denotes the $i^{\text {th }}$ exterior power of the standard representation of $\mathfrak{s u}_{m}$. Since a Cartan subalgebra for $\mathfrak{s u}_{6} \times \mathfrak{s u}_{2}$ is also a Cartan subalgebra for $\mathfrak{e}_{6}$, we can identify the weights of $U_{i}$ as an $\mathfrak{s u}_{6} \times \mathfrak{s u}_{2}$-representation with the weights of $U_{i}$ as an $\mathfrak{e}_{6}$-representation.

Let $u=\frac{1}{2}\left(J_{1}+\cdots+J_{6}\right)+\frac{1}{2}\left(J_{7}+J_{8}\right)$ and $v=\frac{1}{2}\left(J_{1}+\cdots+J_{6}\right)-\frac{1}{2}\left(J_{7}+J_{8}\right)$. One computes that the weights of $U_{1}$ are

$$
\begin{cases}J_{i}+J_{j}-\frac{1}{3}(u+v) & \text { if } \left.1 \leq i<j \leq 6 \quad \text { (corresponding to } \lambda_{6}^{2}\right), \\ J_{i} \pm \frac{1}{2}\left(J_{7}-J_{8}\right)-\frac{5}{6}(u+v) & \text { if } 1 \leq i \leq 6 \quad\left(\text { corresponding to } \lambda_{6}^{5} \otimes \lambda_{2}^{1}\right) .\end{cases}
$$

$W$ preserves these weights. Let $z_{i}=\tau\left(J_{i}\right) \in H^{2}(B S)$ for $1 \leq i \leq 6$ and let $z_{7}=\tau\left(J_{7}-J_{8}\right)$. (As above, by $J_{i}$ we really mean the restriction of the weight for $E_{8}$ to a weight for $E_{7}$.) With respect to this basis, $H^{*}(B S)^{W} \simeq H^{*}\left(B E_{6}\right)$ is generated by

$$
I_{k}=\sum_{1 \leq i<j \leq 6}\left(z_{i}+z_{j}-\frac{1}{3}\left(z_{1}+\cdots+z_{6}\right)\right)^{k}+\sum_{\substack{1 \leq i \leq 6 \\ \epsilon \in\{ \pm 1\}}}\left(z_{i}-\frac{5}{6}\left(z_{1}+\cdots+z_{6}\right)+\frac{1}{2} \epsilon z_{7}\right)^{k} .
$$

As above, $H^{*}(B S)^{W} \simeq \mathbb{Q}\left[I_{2}, I_{5}, I_{6}, I_{8}, I_{9}, I_{12}\right]$, and one computes

$$
I_{2}=5\left(z_{1}^{2}+\cdots+z_{6}^{2}\right)+3 z_{7}^{2}-2 \sum_{1 \leq i<j \leq 6} z_{i} z_{j} .
$$

$\boldsymbol{B F}_{4}$ Let $S \subset F_{4}$ be a maximal torus with Weyl group $W$. Up to conjugation we can assume $S \subset \operatorname{Spin}_{9} \subset F_{4}$ and that $S$ is a maximal torus of $\operatorname{Spin}_{9}$ (see [1, page 53]). This allows us to identify $H^{1}(S) \simeq \mathbb{Q}\left\{L_{1}, \ldots, L_{4}\right\}$ and $H^{2}(B S) \simeq \mathbb{Q}\left[z_{1}, \ldots, z_{4}\right]$. The 26-dimensional fundamental representation of $F_{4}$ has the following nonzero weights (these are the short roots of $F_{4}$; see [1, page 55]):

$$
\begin{cases} \pm L_{i} & \text { if } 1 \leq i \leq 4 \\ \frac{1}{2}\left( \pm L_{1} \pm L_{2} \pm L_{3} \pm L_{4}\right) & \end{cases}
$$

$W$ preserves these weights, and $H^{*}(B S)^{W} \simeq H^{*}\left(B F_{4}\right)$ is generated by

$$
I_{2 k}=\sum_{i=1}^{4} z_{i}^{2 k}+\frac{1}{2^{2 k}} \sum\left(\epsilon_{1} z_{1}+\epsilon_{2} z_{2}+\epsilon_{3} z_{3}+z_{4}\right)^{2 k}
$$


The second sum is over all tuples $\left(\epsilon_{1}, \epsilon_{2}, \epsilon_{3}\right) \in\{ \pm 1\}^{3}$. According to [13, page 1091], $H^{*}(B S)^{W}=\mathbb{Q}\left[I_{2}, I_{6}, I_{8}, I_{12}\right]$. Note that $I_{2}=3\left(z_{1}^{2}+z_{2}^{2}+z_{3}^{2}+z_{4}^{2}\right)$.

$\boldsymbol{B G}_{\mathbf{2}}$ Let $S \subset G_{2}$ be a maximal torus (its dimension is 2). Let $J_{1}, J_{2} \in H^{1}(S)$ be simple roots for $G_{2}$. Denote $z_{i}=\tau\left(J_{i}\right) \in H^{2}(B S)$. The Weyl group $W$ is the dihedral group of order 12 and it permutes the nonzero weights of the 7-dimensional fundamental representation. These weights are

$$
\left\{ \pm J_{1}, \pm\left(J_{1}+J_{2}\right), \pm\left(2 J_{1}+J_{2}\right)\right\} .
$$

See [6, Lecture 22], for example. $H^{*}(B S)^{W}$ is generated the polynomials

$$
I_{2 k}=z_{1}^{2 k}+\left(z_{1}+z_{2}\right)^{2 k}+\left(2 z_{1}+z_{2}\right)^{2 k} \text {. }
$$

According to [13, page 1094], $H^{*}(B S)^{W} \simeq \mathbb{Q}\left[I_{2}, I_{6}\right]$. Note that

$$
I_{2}=2\left(3 z_{1}^{2}+3 z_{1} z_{2}+z_{2}^{2}\right) \text {. }
$$

\section{Computing $\alpha_{1}^{*}: H^{*}\left(B \operatorname{Homeo}\left(S^{n-1}\right)\right) \rightarrow H^{*}(B G)$}

Since the inclusion $K \hookrightarrow G$ is a homotopy equivalence, it induces an isomorphism $H^{*}(B G) \stackrel{\sim}{\rightarrow} H^{*}(B K)$. To understand $\alpha_{1}^{*}$, we study $\left.\alpha_{1}\right|_{K}: K \rightarrow \operatorname{Homeo}\left(S^{n-1}\right)$.

Let $T_{e K}^{1}(G / K)$ be the space of rays through the origin in $T_{e K}(G / K)$. The action of $K$ on $G / K$ induces an action on $T_{e K}^{1}(G / K)$. The exponential map defines a $K$-equivariant homeomorphism

$$
s: T_{e K}^{1}(G / K) \rightarrow \partial(G / K) .
$$

The $K$-action on $T_{e K}(G / K)$ can be described as follows. The adjoint action of $K$ on $\mathfrak{g}=\operatorname{Lie}(G)$ decomposes into invariant subspaces $\mathfrak{k} \oplus \mathfrak{p}$, where $\mathfrak{k}=\operatorname{Lie}(K)$ and $\mathfrak{p} \simeq T_{e K}(G / K)$. This implies the following lemma. For more details, see [18].

Lemma 4.1 The action of $K \subset G$ on $\partial(G / K)$ is induced by a linear representation $\iota: K \rightarrow \operatorname{Aut}(\mathfrak{p})$.

We refer to the representation $\iota: K \rightarrow \operatorname{Aut}(\mathfrak{p}) \simeq \mathrm{GL}_{n}(\mathbb{R})$ as the isotropy representation. Let $r: \mathrm{GL}_{n}(\mathbb{R}) \rightarrow \operatorname{Homeo}\left(S^{n-1}\right)$ be the $\mathrm{GL}_{n}(\mathbb{R})$-action on the space of rays through the origin in $\mathbb{R}^{n}$. By Lemma 4.1, $\left.\alpha_{1}\right|_{K}=r \circ \iota$. Since the map

$$
r^{*}: H^{*}\left(B \operatorname{Homeo}\left(S^{n-1}\right)\right) \rightarrow H^{*}\left(B \mathrm{GL}_{n}(\mathbb{R})\right)
$$

is understood via diagram (3), it remains only to understand

$$
\iota^{*}: H^{*}\left(B \mathrm{GL}_{n}(\mathbb{R})\right) \rightarrow H^{*}(B K) .
$$


As described in Section 3.2, $\iota^{*}$ can be computed using the weights of $\iota$. This will be carried out in Sections 4.1-4.12 as follows. Let $S \subset K$ be a maximal torus. The isotropy representation $\iota: K \rightarrow \operatorname{Aut}(\mathfrak{p})$ is a real representation, so we complexify to get a representation $\iota_{\mathbb{C}}: K_{\mathbb{C}} \rightarrow \operatorname{Aut}\left(\mathfrak{p}_{\mathbb{C}}\right)$. On the maximal abelian subgroup $H \subset K_{\mathbb{C}}$, we obtain weights $\lambda_{i}: H \rightarrow \mathbb{C}^{\times}$. To compute the weights explicitly, we pass to the Lie algebra $\mathfrak{h}$ of $H$ and view the weights as elements of $\mathfrak{h}^{*}=\operatorname{Hom}(\mathfrak{h}, \mathbb{C})$. After computing the weights of $\iota_{\mathbb{C}}$, we use (4) to express the total Chern class $c(\iota \mathbb{C})$ as a polynomial in $H^{*}(B H)$. Finally, since $S \hookrightarrow H$ induces an isomorphism $H^{*}(B H) \rightarrow H^{*}(B S)$, we obtain the Pontryagin classes $p_{i}(\iota)=(-1)^{i} c_{2 i}\left(\iota_{\mathbb{C}}\right)$ as polynomials in $H^{*}(B S)^{W} \simeq H^{*}(B K) \simeq H^{*}(B G)$. This computes $\alpha_{1}^{*}$ since $\alpha_{1}^{*}\left(q_{i}\right)=p_{i}(\iota)$.

Below, $V_{n}$ will denote the standard representation of $\mathfrak{g l}_{n}(\mathbb{C}), \mathfrak{s l}_{n}(\mathbb{C}), \mathfrak{s o}_{n}(\mathbb{C})$ or $\mathfrak{s p}_{2 k}(\mathbb{C})($ for $n=2 k)$.

\subsection{Isotropy representation for $\mathrm{SL}_{n}(\mathbb{R})$}

Upon complexification, we need to study the isotropy representation of $\mathrm{SO}_{n}(\mathbb{C})$, a subgroup of $\mathrm{SL}_{n}(\mathbb{C})$. The adjoint representation of $\mathfrak{s l}_{n}(\mathbb{C})$ is isomorphic to the kernel of the contraction $V_{n} \otimes V_{n}^{*} \rightarrow \mathbb{C}$. As an $\mathfrak{s o}_{n}(\mathbb{C})$-representation,

$$
V_{n} \otimes V_{n}^{*} \simeq \Lambda^{2}\left(V_{n}\right)+\operatorname{Sym}^{2}\left(V_{n}\right) .
$$

$\Lambda^{2}\left(V_{n}\right)$ is the adjoint representation of $\mathfrak{s o}_{n}(\mathbb{C})$. The representation $\operatorname{Sym}^{2}\left(V_{n}\right)$ is not irreducible because $\mathfrak{s o}_{n}(\mathbb{C})$ preserves a symmetric bilinear form $B: \operatorname{Sym}^{2}\left(V_{n}\right) \rightarrow \mathbb{C}$. The kernel of $B$ is the isotropy representation $\mathfrak{p}$.

There is a standard form $B$ for which the diagonal subgroup $H \subset \mathrm{SO}(B) \simeq \mathrm{SO}_{n}(\mathbb{C})$ is a Cartan subgroup (see [6, page 268]) For this choice, we have a standard basis $\mathfrak{h}^{*}=\left\langle L_{1}, \ldots, L_{k}\right\rangle$, where $k=[n / 2]$. If $n$ is even, the weights of $\mathfrak{p}$ are $\pm L_{i} \pm L_{j}$ for $1 \leq i, j \leq k$. If $n$ is odd, we have the additional weights $\pm L_{i}$ for $1 \leq i \leq k$.

As elements of $H^{2}(B H) \simeq \mathbb{Q}\left[y_{1}, \ldots, y_{k}\right]$, we have the following total Chern classes for the isotropy representation. If $n$ is even, then

$$
c(\iota \mathbb{C})=\prod_{i, j}\left(1+y_{i}+y_{j}\right)\left(1+y_{i}-y_{j}\right)\left(1-y_{i}+y_{j}\right)\left(1-y_{i}-y_{j}\right)
$$

and if $n$ is odd, then

$$
(10) c\left(\iota_{\mathbb{C}}\right)=\prod_{i}\left(1-y_{i}\right)\left(1+y_{i}\right) \prod_{i, j}\left(1+y_{i}+y_{j}\right)\left(1+y_{i}-y_{j}\right)\left(1-y_{i}+y_{j}\right)\left(1-y_{i}-y_{j}\right) .
$$




\subsection{Isotropy representation for $\mathrm{SU}_{p, q}$}

Let $n=p+q$. Upon complexification we need to study the isotropy representation of $K_{\mathbb{C}} \subset \mathrm{SL}_{n}(\mathbb{C})$, where $K_{\mathbb{C}}$ is the block diagonal subgroup

$$
K_{\mathbb{C}}=\left(\mathrm{GL}_{p}(\mathbb{C}) \times \mathrm{GL}_{q}(\mathbb{C})\right) \cap \mathrm{SL}_{n}(\mathbb{C}) .
$$

As described in Section 4.1, the adjoint representation of $\mathfrak{s l}_{n}(\mathbb{C})$ is a subspace of $V_{n} \otimes V_{n}^{*}$. As a $\mathfrak{k}_{\mathbb{C}}$-representation,

$$
V_{n} \otimes V_{n}^{*}=\left(V_{p} \otimes V_{p}^{*}+V_{q} \otimes V_{q}^{*}\right)+\left(V_{p} \otimes V_{q}^{*}+V_{p}^{*} \otimes V_{q}\right) .
$$

The adjoint representation of $\mathfrak{k}_{\mathbb{C}}$ is a codimension- 1 subspace of the first summand. The second summand is the isotropy representation $\mathfrak{p}$.

The diagonal subgroup $H \subset K_{\mathbb{C}}$ coincides with the diagonal subgroup of $\operatorname{SL}_{n}(\mathbb{C})$, so we identify $\mathfrak{h}^{*}$ as the quotient of $\left\langle L_{1}, \ldots, L_{n}\right\rangle$ by the subspace generated by $L_{1}+\cdots+L_{n}$. The weights of the isotropy representation are $\pm\left(L_{i}-L_{j}\right)$ for $1 \leq i \leq p$ and $p+1 \leq j \leq p+q$.

As an element of $H^{2}(B H) \subset \mathbb{Q}\left[y_{1} \ldots, y_{p}, z_{1}, \ldots, z_{q}\right]$, the total Chern class for the complexified isotropy representation is

$$
c\left(\iota_{\mathbb{C}}\right)=\prod_{\substack{1 \leq i \leq p \\ 1 \leq j \leq q}}\left(1+\left(y_{i}-z_{j}\right)\right)\left(1-\left(y_{i}-z_{j}\right)\right)=\prod_{\substack{1 \leq i \leq p \\ 1 \leq j \leq q}}\left(1-\left(y_{i}-z_{j}\right)^{2}\right) .
$$

\subsection{Isotropy representation for $\operatorname{Sp}_{2 n}(\mathbb{R})$}

Upon complexification we need to study the isotropy representation of the subgroup $\mathrm{GL}_{n}(\mathbb{C}) \subset \mathrm{Sp}_{2 n}(\mathbb{C})$ of matrices of the form

$$
\left(\begin{array}{ll}
A & \\
& \left(A^{t}\right)^{-1}
\end{array}\right)
$$

for $A \in \mathrm{GL}_{n}(\mathbb{C})$.

As a $\mathfrak{g l}_{n}(\mathbb{C})$-representation, the standard representation of $\mathfrak{s p}_{2 n}(\mathbb{C})$ decomposes as $V_{2 n}=V_{n}+V_{n}^{*}$. The adjoint representation of $\mathfrak{s p}_{2 n}(\mathbb{C})$ is isomorphic to $\operatorname{Sym}^{2}(V)$ and, as a $\mathfrak{g l}_{n}(\mathbb{C})$-representation,

$$
\operatorname{Sym}^{2}(V) \simeq\left(V_{n} \otimes V_{n}^{*}\right)+\left(\operatorname{Sym}^{2}\left(V_{n}\right)+\operatorname{Sym}^{2}\left(V_{n}^{*}\right)\right) .
$$

$V_{n} \otimes V_{n}^{*}$ is the adjoint representation of $\mathfrak{g l}_{n}(\mathbb{C})$ and $\operatorname{Sym}^{2}\left(V_{n}\right)+\operatorname{Sym}^{2}\left(V_{n}^{*}\right)$ is the isotropy representation. 
Let $H \subset \mathrm{GL}_{n}(\mathbb{C})$ be the diagonal subgroup and identify $\mathfrak{h}^{*}=\left\langle L_{1}, \ldots, L_{n}\right\rangle$ using the standard basis. The weights of the isotropy representation are $\pm\left(L_{i}+L_{j}\right)$ for $1 \leq i, j \leq n$. As an element of $H^{2}(B H) \simeq \mathbb{Q}\left[y_{1}, \ldots, y_{n}\right]$, the total Chern class of the complexified isotropy representation is

$$
\begin{aligned}
c\left(\iota_{\mathbb{C}}\right) & =\prod_{i<j}\left(1+y_{i}+y_{j}\right)\left(1-\left(y_{i}+y_{j}\right)\right) \prod_{i}\left(1+2 y_{i}\right)\left(1-2 y_{i}\right) \\
& =\prod_{i<j}\left(1-\left(y_{i}+y_{j}\right)^{2}\right) \prod_{i}\left(1-4 y_{i}^{2}\right) .
\end{aligned}
$$

\subsection{Isotropy representation for $\mathrm{SO}_{p, q}$}

Let $n=p+q, a=[p / 2]$ and $b=[q / 2]$. Upon complexification, we need to study the isotropy representation of $\mathrm{SO}_{p}(\mathbb{C}) \times \mathrm{SO}_{q}(\mathbb{C}) \subset \mathrm{SO}_{n}(\mathbb{C})$.

The adjoint representation of $\mathfrak{s o}_{n}(\mathbb{C})$ is $\Lambda^{2}\left(V_{n}\right)$ and, as an $\mathfrak{s o}_{p}(\mathbb{C}) \times \mathfrak{s o}_{q}(\mathbb{C})$-representation,

$$
\Lambda^{2}\left(V_{n}\right) \simeq\left(\Lambda^{2}\left(V_{p}\right)+\Lambda^{2}\left(V_{q}\right)\right)+V_{p} \otimes V_{q} .
$$

$\Lambda^{2}\left(V_{p}\right)+\Lambda^{2}\left(V_{q}\right)$ is the adjoint representation of $\mathfrak{s o}_{p}(\mathbb{C}) \times \mathfrak{s o}_{q}(\mathbb{C})$, and $V_{p} \otimes V_{q}$ is the isotropy representation.

Let $H \subset \mathrm{SO}_{p}(\mathbb{C}) \times \mathrm{SO}_{q}(\mathbb{C})$ be the standard Cartan subgroup, and identify $\mathfrak{h}^{*}=$ $\left\langle L_{1}, \ldots, L_{a}, L_{a+1}, \ldots, L_{a+b}\right\rangle$. In this basis, the weights of the isotropy representation are

$$
\begin{cases} \pm L_{i} \pm L_{j} & \text { if } p, q \text { are even, } \\ \pm L_{i} \pm L_{j}, \pm L_{i} & \text { if } p \text { is even and } q \text { is odd } \\ \pm L_{i} \pm L_{j}, \pm L_{j} & \text { if } p \text { is odd and } q \text { is even } \\ \pm L_{i} \pm L_{j}, \pm L_{i}, \pm L_{j} & \text { if } p \text { is odd and } q \text { is odd }\end{cases}
$$

where $1 \leq i \leq a$ and $a+1 \leq j \leq a+b$.

As an element of $H^{*}(B H) \simeq \mathbb{Q}\left[y_{1}, \ldots, y_{a}, z_{1}, \ldots, z_{b}\right]$, we have the following total Chern classes. If $p$ and $q$ are both even, then

$$
\begin{aligned}
c\left(\iota_{\mathbb{C}}\right) & =\prod\left(1+y_{i}+z_{j}\right)\left(1+y_{i}-z_{j}\right)\left(1-y_{i}+z_{j}\right)\left(1-y_{i}-z_{j}\right) \\
& =\prod_{\substack{1 \leq i \leq a \\
1 \leq j \leq b}}\left(1-\left(y_{i}+z_{j}\right)^{2}\right)\left(1-\left(y_{i}-z_{j}\right)^{2}\right) .
\end{aligned}
$$

If $p$ is even and $q$ is odd, then

$$
c\left(\iota_{\mathbb{C}}\right)=\prod_{i=1}^{a}\left(1-y_{i}^{2}\right) \prod_{\substack{1 \leq i \leq a \\ 1 \leq j \leq b}}\left(1-\left(y_{i}+z_{j}\right)^{2}\right)\left(1-\left(y_{i}-z_{j}\right)^{2}\right) .
$$


If $p$ is odd and $q$ is even, then

$$
c\left(\iota_{\mathbb{C}}\right)=\prod_{i=1}^{b}\left(1-z_{j}^{2}\right) \prod_{\substack{1 \leq i \leq a \\ 1 \leq j \leq b}}\left(1-\left(y_{i}+z_{j}\right)^{2}\right)\left(1-\left(y_{i}-z_{j}\right)^{2}\right) .
$$

If $p$ and $q$ are both odd, then

$$
c\left(\iota_{\mathbb{C}}\right)=\prod_{i=1}^{a}\left(1-y_{i}^{2}\right) \prod_{j=1}^{b}\left(1-z_{j}^{2}\right) \prod_{\substack{1 \leq i \leq a \\ 1 \leq j \leq b}}\left(1-\left(y_{i}+z_{j}\right)^{2}\right)\left(1-\left(y_{i}-z_{j}\right)^{2}\right) .
$$

\subsection{Isotropy representation for $\mathrm{Sp}_{p, q}$}

Let $n=p+q$. After complexifying, we need to study the isotropy representation of $\mathrm{Sp}_{2 p}(\mathbb{C}) \times \mathrm{Sp}_{2 q}(\mathbb{C}) \subset \mathrm{Sp}_{2 n}(\mathbb{C})$.

The adjoint representation of $\mathfrak{s p}_{2 n}(\mathbb{C})$ is $\operatorname{Sym}^{2}\left(V_{2 n}\right)$ and, as an $\mathfrak{s p}_{2 p}(\mathbb{C}) \times \mathfrak{s p}_{2 q}(\mathbb{C})$ representation,

$$
\operatorname{Sym}^{2}\left(V_{2 n}\right) \simeq\left(\operatorname{Sym}^{2}\left(V_{2 p}\right)+\operatorname{Sym}^{2}\left(V_{2 q}\right)\right)+V_{2 p} \otimes V_{2 q} .
$$

$\operatorname{Sym}^{2}\left(V_{2 p}\right)+\operatorname{Sym}^{2}\left(V_{2 q}\right)$ is the adjoint representation of $\mathfrak{s p}_{2 p}(\mathbb{C}) \times \mathfrak{s p}_{2 q}(\mathbb{C})$, and $V_{2 p} \otimes V_{2 q}$ is the isotropy representation.

Let $H \subset \mathrm{Sp}_{2 p}(\mathbb{C}) \times \mathrm{Sp}_{2 q}(\mathbb{C})$ be the standard Cartan subgroup, and identify $\mathfrak{h}^{*}=$ $\left\langle L_{1}, \ldots, L_{p}, L_{p+1}, \ldots, L_{p+q}\right\rangle$. The weights of the isotropy representation are $\pm L_{i} \pm L_{j}$, where $1 \leq i \leq p$ and $2 p+1 \leq j \leq 2 p+q$. As an element of $H^{2}(B H) \simeq$ $\mathbb{Q}\left[y_{1}, \ldots, y_{p}, z_{1}, \ldots, z_{q}\right]$, the total Chern class of the complexified isotropy representation is

$$
c\left(\iota_{\mathbb{C}}\right)=\prod_{\substack{1 \leq i \leq p \\ 1 \leq j \leq q}}\left(1+y_{i}+z_{j}\right)\left(1+y_{i}-z_{j}\right)\left(1-y_{i}+z_{j}\right)\left(1-y_{i}-z_{j}\right) .
$$

\subsection{Isotropy representation for $\mathrm{SO}_{2 n}^{*}$}

Define $\mathrm{SO}_{2 n}^{*}$ as the subgroup of $\mathrm{GL}_{2 n}(\mathbb{C})$ that preserves the Hermitian form and bilinear form defined by

$$
I_{n, n}=\left(\begin{array}{cc}
I_{n} & \\
& -I_{n}
\end{array}\right) \quad \text { and } \quad B_{n}=\left(\begin{array}{cc}
0 & I_{n} \\
I_{n} & 0
\end{array}\right),
$$


respectively. We complexify and study the isotropy representation of the subgroup $\mathrm{GL}_{n}(\mathbb{C}) \subset \mathrm{SO}_{2 n}(\mathbb{C})$ of matrices of the form

$$
\left(\begin{array}{ll}
A & \\
& \left(A^{t}\right)^{-1}
\end{array}\right)
$$

for $A \in \mathrm{GL}_{n}(\mathbb{C})$.

As a $\mathfrak{g l}_{n}(\mathbb{C})$-representation, the standard representation of $\mathfrak{s o}_{2 n}(\mathbb{C})$ decomposes as $V_{2 n}=V_{n}+V_{n}^{*}$. The adjoint representation of $\mathfrak{s o}_{2 n}(\mathbb{C})$ is $\Lambda^{2}\left(V_{2 n}\right)$ and, as a $\mathfrak{g l}_{n}(\mathbb{C})$ representation,

$$
\Lambda^{2}\left(V_{2 n}\right) \simeq V_{n} \otimes V_{n}^{*}+\left(\Lambda^{2}\left(V_{n}\right)+\Lambda^{2}\left(V_{n}^{*}\right)\right) .
$$

$V_{n} \otimes V_{n}^{*}$ is the adjoint representation of $\mathfrak{g l}_{n}(\mathbb{C})$, and $\Lambda^{2}\left(V_{n}\right)+\Lambda^{2}\left(V_{n}^{*}\right)$ is the isotropy representation.

Let $H \subset \mathrm{GL}_{n}(\mathbb{C})$ be the diagonal subgroup and identify $\mathfrak{h}^{*}=\left\langle L_{1}, \ldots, L_{n}\right\rangle$. The weights of the isotropy representation are $\pm\left(L_{i}+L_{j}\right)$ for $1 \leq i<j \leq n$. As an element of $H^{2}(B H) \simeq \mathbb{Q}\left[y_{1}, \ldots, y_{n}\right]$, the total Chern class is

$$
c(\iota \mathbb{C})=\prod_{i<j}\left(1+\left(y_{i}+y_{j}\right)\right)\left(1-\left(y_{i}+y_{j}\right)\right)=\prod_{i<j}\left(1-\left(y_{i}+y_{j}\right)^{2}\right) .
$$

\subsection{Isotropy representation for $\mathrm{SU}_{2 n}^{*}$}

After complexifying, we need to study the isotropy representation of $\operatorname{Sp}_{2 n}(\mathbb{C})$, a subgroup of $\mathrm{SL}_{2 n}(\mathbb{C})$. As described in Section 4.1, the adjoint representation of $\mathfrak{s l}_{2 n}(\mathbb{C})$ is contained in $V_{2 n} \otimes V_{2 n}^{*}$. As a $\mathfrak{s p}_{2 n}(\mathbb{C})$-representation,

$$
V_{2 n} \otimes V_{2 n}^{*} \simeq \operatorname{Sym}^{2}\left(V_{2 n}\right)+\Lambda^{2}\left(V_{2 n}\right) .
$$

$\operatorname{Sym}^{2}\left(V_{2 n}\right)$ is the adjoint representation of $\mathfrak{s p}_{2 n}(\mathbb{C})$. The representation $\Lambda^{2}\left(V_{2 n}\right)$ is not irreducible because, by definition, $\mathfrak{s p}_{2 n}(\mathbb{C})$ preserves an antisymmetric bilinear form $J: \Lambda^{2}\left(V_{2 n}\right) \rightarrow \mathbb{C}$. The kernel of $J$ is the isotropy representation $\mathfrak{p}$.

Let $H \subset \mathrm{Sp}_{2 n}(\mathbb{C})$ be the standard Cartan subgroup, and identify $\mathfrak{h}^{*}=\left\langle L_{1}, \ldots, L_{n}\right\rangle$. The nonzero weights of the isotropy representation are $\pm L_{i} \pm L_{j}$ for $1 \leq i<j \leq n$. As an element of $H^{2}(B H)$, the total Chern class of the isotropy representation is

$$
c\left(\iota_{\mathbb{C}}\right)=\prod_{1 \leq i<j \leq n}\left(1+y_{i}+y_{j}\right)\left(1+y_{i}-y_{j}\right)\left(1-y_{i}+y_{j}\right)\left(1-y_{i}-y_{j}\right) .
$$




\subsection{Isotropy representation for real forms of $E_{8}$}

Let $\mathfrak{h}^{*}\left(\mathfrak{e}_{8}\right)$ be the dual to the Cartan subalgebra of $\mathfrak{e}_{8}$. As in [1, page 56], we identify $\mathfrak{h}_{8}^{*}=\left\langle J_{1}, \ldots, J_{8}\right\rangle$.

In the remainder of this section, we use $\lambda_{n}^{i}$ to denote the $i^{\text {th }}$ exterior power of the standard representations of the real Lie algebras $\mathfrak{s u}_{n}, \mathfrak{s o}_{n}$ and $\mathfrak{s p}_{n}$.

$\boldsymbol{E}_{\mathbf{8}(\mathbf{8})}$ The maximal compact subgroup $K \subset E_{8(8)}$ has Lie algebra $\mathfrak{s o}_{16}$. As a $K$-representation, $\mathfrak{e}_{8}$ decomposes as

$$
\mathfrak{e}_{8}=\mathfrak{s o}_{16} \oplus \Delta^{+},
$$

where $\Delta^{+}$is the positive spin representation of $\mathfrak{s o}_{16}$ (see [1, Chapter 6]). Then the isotropy representation of $K \subset E_{8}$ is the spin representation. Let $\mathfrak{h}^{*}\left(\mathfrak{s o}_{16}\right)$ denote the dual to the Cartan subalgebra of $\mathfrak{s o}_{16}$. We identify $\mathfrak{h}^{*}\left(\mathfrak{s o}_{16}\right)=\left\langle L_{1}, \ldots, L_{8}\right\rangle$. The weights of the isotropy representation are

$$
\frac{1}{2}\left( \pm L_{1} \pm L_{2} \pm \cdots \pm L_{8}\right)
$$

where the number of + signs is even.

Let $S \subset K$ be a maximal torus. As an element of $H^{*}(B S) \simeq \mathbb{Q}\left[y_{1}, \ldots, y_{8}\right]$, the total Chern class of the complexified isotropy representation is

$$
c\left(\iota_{\mathbb{C}}\right)=\prod\left(1-\frac{1}{2}\left(\epsilon_{1} y_{1}+\cdots+\epsilon_{8} y_{8}\right)\right),
$$

where the product is over all tuples $\left(\epsilon_{1}, \ldots, \epsilon_{8}\right) \in\{ \pm 1\}^{8}$ that have an even number of negatives.

$\boldsymbol{E}_{\mathbf{8}(-\mathbf{2 4})}$ The maximal compact subgroup $K \subset E_{8(-24)}$ has Lie algebra $\mathfrak{e}_{7} \times \mathfrak{s u}{ }_{2}$. As a $K$-representation, $\mathfrak{e}_{8}$ decomposes as

$$
\mathfrak{e}_{8}=\left(\mathfrak{e}_{7} \oplus \mathfrak{s u}_{2}\right) \oplus\left(V \otimes \lambda_{2}^{1}\right),
$$

where $V$ is the 56-dimensional representation of $E_{7}$. See [1, page 54].

Since $K \subset E_{8}$ have the same rank, we can identify the (dual) Cartan subalgebras $\mathfrak{h}^{*}\left(\mathfrak{e}_{8}\right)=\left\langle J_{1}, \ldots, J_{8}\right\rangle$ and $\mathfrak{h}^{*}(K)=\left\langle J_{1}, \ldots, J_{6}, \frac{1}{2}\left(J_{7}+J_{8}\right)\right\rangle \oplus\left\langle\frac{1}{2}\left(J_{7}-J_{8}\right)\right\rangle$.

The roots for $\mathfrak{e}_{7} \times \mathfrak{s u}_{2}$ are $\pm\left(J_{7}-J_{8}\right)$ together with all the roots of $\mathfrak{e}_{8}$ that are orthogonal to $J_{7}-J_{8}$. The roots of $\mathfrak{e}_{8}$ that are not roots of $\mathfrak{e}_{7} \times \mathfrak{s u}_{2}$ correspond to the weights of the isotropy representation $\mathfrak{p}=V \otimes \lambda_{2}^{1}$. Then the weights of $\mathfrak{p}$ are

$$
\begin{cases} \pm J_{i} \pm \frac{1}{2}\left(J_{7}+J_{8}\right) \pm \frac{1}{2}\left(J_{7}-J_{8}\right) & \text { if } 1 \leq i \leq 6 \\ \frac{1}{2}\left( \pm J_{1} \pm \cdots \pm J_{6}\right) \pm \frac{1}{2}\left(J_{7}-J_{8}\right) & \text { if an even number of the } J_{i} \text { are negated. }\end{cases}
$$


Let $S \subset K$ be a maximal torus. Define $y_{i}=\tau\left(J_{i}\right) \in H^{2}(B S)$ for $1 \leq i \leq 6$, $y_{7}=\tau\left(J_{7}+J_{8}\right)$ and $y_{8}=\tau\left(J_{7}-J_{8}\right)$. The total Chern class for the complexified isotropy representation $c\left(\iota_{\mathbb{C}}\right) \in H^{*}(B S)^{W}$ is

(21) $c\left(\iota_{\mathbb{C}}\right)=\prod\left(1-\left(\epsilon y_{i}+\epsilon_{7} \cdot \frac{1}{2} y_{7}+\epsilon_{8} \cdot \frac{1}{2} y_{8}\right)\right) \prod\left(1-\frac{1}{2}\left(\epsilon_{1} y_{1}+\cdots+\epsilon_{6} y_{6}+\epsilon_{8} y_{8}\right)\right)$.

The first product is over $i=1, \ldots, 6$ and all tuples $\left(\epsilon, \epsilon_{7}, \epsilon_{8}\right) \in\{ \pm 1\}^{3}$. The second product is over the tuples $\left(\epsilon_{1}, \ldots, \epsilon_{6}, \epsilon_{8}\right) \in\{ \pm 1\}^{7}$ with an even number of negatives.

\subsection{Isotropy representation for real forms of $E_{7}$}

$\boldsymbol{E}_{\mathbf{7 ( 7 )}}$ The maximal compact subgroup $K \subset E_{7(7)}$ has Lie algebra $\mathfrak{s u}_{8}$. Following [1, page 69], as a $K$-representation, $\mathfrak{e}_{7}$ decomposes as

$$
\mathfrak{e}_{7}=\mathfrak{s u}_{8} \oplus \lambda_{8}^{4} .
$$

Here $\mathfrak{p}=\lambda_{8}^{4}$ is the isotropy representation, and the weights are $L_{i_{1}}+L_{i_{2}}+L_{i_{3}}+L_{i_{4}}$ for $1 \leq i_{1}<\cdots<i_{4} \leq 8$.

Let $S \subset K$ be a maximal torus. We identify $H^{*}(B S)$ with the quotient of $\mathbb{Q}\left[y_{1}, \ldots, y_{8}\right]$ by the ideal generated by $y_{1}+\cdots+y_{8}$. The total Chern class of the complexified isotropy representation is

$$
c\left(\iota_{\mathbb{C}}\right)=\prod_{1 \leq i_{1}<i_{2}<i_{3}<i_{4} \leq 8}\left(1-\left(y_{i_{1}}+y_{i_{2}}+y_{i_{3}}+y_{i_{4}}\right)\right) .
$$

$\boldsymbol{E}_{\mathbf{7 ( - 5 )}}$ The maximal compact subgroup $K \subset E_{7(-5)}$ has Lie algebra $\mathfrak{s o}_{12} \times \mathfrak{s u}_{2}$. Following [1, page 52], $\mathfrak{e}_{7}$ decomposes as a $K$-representation as

$$
\mathfrak{e}_{7}=\left(\mathfrak{s o}_{12} \oplus \mathfrak{s u}_{2}\right) \oplus \Delta^{+} \otimes \lambda_{2}^{1},
$$

where $\Delta^{+}$is the positive spin representation of $K$. Here $\mathfrak{p}=\Delta^{+} \otimes \lambda^{1}$ is the isotropy representation. Identify $\mathfrak{h}^{*}(K) \simeq\left\langle L_{1}, \ldots, L_{6}\right\rangle \oplus\left\langle L_{7}\right\rangle$ in the standard way. The weights of the isotropy representation are $\frac{1}{2}\left(\epsilon_{1} L_{1}+\cdots+\epsilon_{6} L_{6}\right) \pm L_{7}$, where $\left(\epsilon_{1}, \ldots, \epsilon_{6}\right) \in\{ \pm 1\}^{6}$ has an even number of negatives.

Let $S \subset K$ be a maximal torus. As an element of $H^{*}(B S) \simeq \mathbb{Q}\left[y_{1}, \ldots, y_{6}\right] \otimes \mathbb{Q}\left[y_{7}\right]$, the total Chern class for the complexified isotropy representation is

$$
c\left(\iota_{\mathbb{C}}\right)=\prod\left(1-\frac{1}{4}\left(\epsilon_{1} y_{1}+\cdots+\epsilon_{6} y_{6}+y_{7}\right)^{2}\right),
$$

where $\left(\epsilon_{1}, \ldots, \epsilon_{6}\right) \in\{ \pm 1\}^{6}$ has an even number of negatives. 
$\boldsymbol{E}_{\mathbf{7 ( - 2 5 )}}$ The maximal compact subgroup $K \subset E_{7(-25)}$ has Lie algebra $\mathfrak{e}_{6} \times \mathfrak{s o}_{2}$. In a similar way to [1, page 52], one shows that $\mathfrak{e}_{7}$ decomposes as a $K$-representation:

$$
\mathfrak{e}_{7}=\left(\mathfrak{e}_{6} \oplus \mathfrak{s o}_{2}\right) \oplus U_{1} \otimes \xi^{2} \oplus U_{2} \otimes \xi^{-2},
$$

where $U_{1}$ and $U_{2}$ are the 27-dimensional representations of $E_{6}$. Make the identifications

$$
\mathfrak{h}^{*}\left(\mathfrak{e}_{6}\right)=\left\langle J_{1}, \ldots, J_{5}, J_{6}+J_{7}+J_{8}\right\rangle \quad \text { and } \quad \mathfrak{h}^{*}\left(\mathfrak{e}_{7}\right)=\left\langle J_{1}, \ldots, J_{6}, J_{7}+J_{8}\right\rangle
$$

as subspaces of $h^{*}\left(\mathfrak{e}_{8}\right)$ (see Section 3). The orthogonal complement of $\mathfrak{h}^{*}\left(\mathfrak{e}_{6}\right)$ in $\mathfrak{h}^{*}\left(\mathfrak{e}_{7}\right)$ is 1 -dimensional and generated by $-2 J_{6}+J_{7}+J_{8}$. We have

$$
\mathfrak{h}^{*}\left(\mathfrak{e}_{6} \times \mathfrak{s o}_{2}\right)=\left\langle J_{1}, \ldots, J_{5}, J_{6}+J_{7}+J_{8}\right\rangle \oplus\left\langle-2 J_{6}+J_{7}+J_{8}\right\rangle .
$$

The weights of the isotropy representation are the roots of $\mathfrak{e}_{7}$ that are not roots of $\mathfrak{e}_{6} \times \mathfrak{s o}_{2}$. These are $\pm\left(J_{7}+J_{8}\right), \pm J_{i} \pm J_{6}$ for $1 \leq i \leq 5$, and

$$
\frac{1}{2}\left(\epsilon_{1} J_{1}+\cdots+\epsilon_{5} J_{5}+\epsilon_{6}\left(J_{6}-J_{7}-J_{8}\right)\right),
$$

where $\left(\epsilon_{1}, \ldots, \epsilon_{6}\right) \in\{ \pm 1\}^{6}$ has even number of negatives. To write these roots as weights of $\mathfrak{e}_{6} \times \mathfrak{s o}_{2}$, note that

$$
J_{7}+J_{8}=\frac{1}{3}\left[2\left(J_{6}+J_{7}+J_{8}\right)+\left(-2 J_{6}+J_{7}+J_{8}\right)\right]
$$

and

$$
J_{6}=\frac{1}{3}\left[\left(J_{6}+J_{7}+J_{8}\right)-\left(-2 J_{6}+J_{7}+J_{8}\right)\right] .
$$

The weights of the isotropy representation are

- $\quad \pm \frac{1}{3}\left[2\left(J_{6}+J_{7}+J_{8}\right)+\left(-2 J_{6}+J_{7}+J_{8}\right)\right]$,

- $\pm J_{i} \pm \frac{1}{3}\left[\left(J_{6}+J_{7}+J_{8}\right)-\left(-2 J_{6}+J_{7}+J_{8}\right)\right]$ for $1 \leq i \leq 5$, and

- $\frac{1}{2}\left(\epsilon_{1} J_{1}+\cdots+\epsilon_{5} J_{5}+\epsilon_{6} \cdot \frac{1}{3}\left[-\left(J_{6}+J_{7}+J_{8}\right)-2\left(-2 J_{6}+J_{7}+J_{8}\right)\right]\right)$ for $\left(\epsilon_{1}, \ldots, \epsilon_{6}\right) \in\{ \pm 1\}^{6}$ with an even number of negatives.

Let $S \subset E_{6} \times \mathrm{SO}_{2}$ be a maximal torus. Let $z_{i}=\tau\left(J_{i}\right) \in H^{2}(B S)$ for $1 \leq i \leq 5$, and let $z_{6}=\tau\left(J_{6}+J_{7}+J_{8}\right)$ and $z_{7}=\tau\left(-2 J_{6}+J_{7}+J_{8}\right)$. As elements of $H^{2}(B S)$, the weights of the isotropy representation are

- $\pm\left(2 z_{6}+z_{7}\right)$,

- $\pm z_{i} \pm \frac{1}{3}\left(z_{6}-z_{7}\right)$ for $1 \leq i \leq 5$, and

- $\frac{1}{2}\left(\epsilon_{1} z_{1}+\cdots+\epsilon_{5} z_{5}+\epsilon_{6} \cdot \frac{1}{3}\left(-z_{6}-2 z_{7}\right)\right)$ for $\left(\epsilon_{1}, \ldots, \epsilon_{6}\right) \in\{ \pm 1\}^{6}$ with even number of negatives. 
The total Chern class is

$$
\begin{aligned}
c\left(\iota_{\mathbb{C}}\right)=\left(1-\frac{1}{9}\left(2 z_{6}+z_{7}\right)^{2}\right) & \prod\left(1-\left(z_{i}+\frac{1}{3}\left(z_{6}-z_{7}\right)\right)^{2}\right)\left(1-\left(z_{i}-\frac{1}{3}\left(z_{6}-z_{7}\right)\right)^{2}\right) \\
\times & \times\left(1-\frac{1}{4}\left(\epsilon_{1} z_{1}+\cdots+\epsilon_{5} z_{5}+\epsilon_{6} \frac{1}{3}\left(-z_{6}-2 z_{7}\right)\right)^{2}\right) .
\end{aligned}
$$

The first product is over $1 \leq i \leq 5$. The second product is over $\left(\epsilon_{1}, \ldots, \epsilon_{6}\right) \in\{ \pm 1\}^{6}$ with an even number of negatives.

\subsection{Isotropy representation for real forms of $E_{6}$}

$\boldsymbol{E}_{\mathbf{6}(6)}$ The maximal compact subgroup $K \subset E_{6(6)}$ has Lie algebra $\mathfrak{s p}_{4}$. As a $K-$ representation, $\mathfrak{e}_{6}$ decomposes as

$$
\mathfrak{e}_{6}=\mathfrak{s p}_{4} \oplus W,
$$

where $W \subset \lambda_{8}^{4}$ is the kernel of the contraction map $\lambda_{8}^{4} \rightarrow \lambda_{8}^{2}$. It is the irreducible representation of $K$ with highest weight $L_{1}+L_{2}+L_{3}+L_{4}$. So $\mathfrak{p}=W$ and the nonzero weights of the isotropy representation are

$$
\begin{cases} \pm L_{i} \pm L_{j} & \text { if } 1 \leq i<j \leq 4 \\ \epsilon_{1} L_{1}+\cdots+\epsilon_{4} L_{4} & \text { if }\left(\epsilon_{1}, \ldots, \epsilon_{4}\right) \in\{ \pm 1\}^{4} .\end{cases}
$$

Let $S \subset \mathrm{Sp}_{4}$ be a maximal torus. As an element of $H^{*}(B S) \simeq \mathbb{Q}\left[y_{1}, \ldots, y_{4}\right]$, the total Chern class is

$$
\begin{aligned}
c\left(\iota_{\mathbb{C}}\right)=\prod_{1 \leq i<j \leq 4}\left(1-\left(y_{i}+y_{j}\right)^{2}\right)(1- & \left.\left(y_{i}-y_{j}\right)^{2}\right) \\
& \times \prod\left(1-\left(\epsilon_{1} y_{1}+\epsilon_{2} y_{2}+\epsilon_{3} y_{3}+y_{4}\right)^{2}\right) .
\end{aligned}
$$

The second product is over all tuples $\left(\epsilon_{1}, \epsilon_{2}, \epsilon_{3}\right) \in\{ \pm 1\}^{3}$.

$\boldsymbol{E}_{\mathbf{6}(2)}$ The maximal compact subgroup $K \subset E_{6(2)}$ has Lie algebra $\mathfrak{s u}_{6} \times \mathfrak{s u}_{2}$. As a $K$-representation, $\mathfrak{e}_{6}$ decomposes as

$$
\mathfrak{e}_{6}=\left(\mathfrak{s u}_{6} \oplus \mathfrak{s u}_{2}\right) \oplus\left(\lambda_{6}^{3} \otimes \lambda \lambda_{2}^{1}\right) .
$$

With respect to the standard basis for $\mathfrak{h}^{*}\left(\mathfrak{s u}_{6} \times \mathfrak{s u}_{2}\right)$, the isotropy representation $\mathfrak{p}=\lambda_{6}^{3} \otimes \lambda_{2}^{1}$ has weights $L_{i_{1}}+L_{i_{2}}+L_{i_{3}} \pm L_{7}$, where $1 \leq i_{1}<i_{2}<i_{3} \leq 6$.

Let $S \subset K$ be a maximal torus. As an element of $\mathbb{Q}\left[y_{1}, \ldots, y_{7}\right] \rightarrow H^{*}(B S)$ the total Chern class is

$$
c(\iota \mathbb{C})=\prod_{1 \leq i_{1}<i_{2}<i_{3} \leq 6}\left(1-\left(y_{i_{1}}+y_{i_{2}}+y_{i_{3}}+y_{7}\right)^{2}\right) .
$$


$\boldsymbol{E}_{\mathbf{6}(-14)}$ The maximal compact subgroup $K \subset E_{6(-14)}$ has Lie algebra $\mathfrak{s o}_{10} \times \mathfrak{s o}_{2}$. According to [1, page 53], as a $K$-representation $\mathfrak{e}_{6}$ decomposes as

$$
\mathfrak{e}_{6}=\left(\mathfrak{s o}_{10}+\mathfrak{s o}_{2}\right) \oplus\left(\Delta^{+} \otimes \xi^{3}+\Delta^{-} \otimes \xi^{-3}\right),
$$

where $\xi: \mathrm{SO}_{2} \rightarrow \mathbb{C}^{\times}$denotes the identity representation of $\mathrm{SO}_{2}$ (or rather the induced Lie algebra representation), and $\xi^{k}: \mathrm{SO}_{2} \rightarrow \mathbb{C}^{\times}$denotes the $k^{\text {th }}$ power of $\xi$.

With respect to the standard basis $\mathfrak{h}^{*}\left(\mathfrak{s o}_{10} \times \mathfrak{s o}_{2}\right)=\left\langle L_{1}, \ldots, L_{5}\right\rangle \oplus\left\langle L_{6}\right\rangle$, the isotropy representation $\mathfrak{p}=\Delta^{+} \otimes \xi^{3}+\Delta^{-} \otimes \xi^{-3}$ has weights

$$
\frac{1}{2}\left(\epsilon_{1} L_{1}+\cdots+\epsilon_{5} L_{5}\right)+3 \epsilon_{6} L_{6},
$$

where $\left(\epsilon_{1}, \cdots, \epsilon_{6}\right) \in\{ \pm 1\}^{6}$ has an even number of negatives.

Let $S \subset K$ be a maximal torus. As an element of $H^{*}(B S) \simeq \mathbb{Q}\left[y_{1}, \ldots, y_{5}\right] \otimes \mathbb{Q}\left[y_{6}\right]$, the total Chern class of the isotropy representation is

$$
c\left(\iota_{\mathbb{C}}\right)=\prod\left(1-\frac{1}{4}\left(\epsilon_{1} y_{1}+\cdots+\epsilon_{5} y_{5}+6 y_{6}\right)^{2}\right),
$$

with the product over the tuples $\left(\epsilon_{1}, \ldots, \epsilon_{5}\right) \in\{ \pm 1\}^{5}$ with an even number of negatives. $\boldsymbol{E}_{\mathbf{6}(-26)}$ The maximal compact subgroup $K \subset E_{6(-26)}$ has Lie algebra $\mathfrak{f}_{4}$. Following [1, page 95], as a $K$-representation, $\mathfrak{e}_{6}$ decomposes as

$$
\mathfrak{e}_{6}=\mathfrak{f}_{4} \oplus U,
$$

where $U$ is the 26-dimensional fundamental representation of $\mathfrak{f}_{4}$. With respect to the standard basis $\mathfrak{h}^{*}\left(\mathfrak{f}_{4}\right)=\left\langle L_{1}, \ldots, L_{4}\right\rangle$, the isotropy representation $\mathfrak{p} \simeq U$ has weights $\pm L_{i}$ for $1 \leq i \leq 4$ and $\frac{1}{2}\left( \pm L_{1} \pm L_{2} \pm L_{3} \pm L_{4}\right)$.

Let $S \subset F_{4}$ be a maximal torus. As an element of $H^{*}(B S) \simeq \mathbb{Q}\left[y_{1}, y_{2}, y_{3}, y_{4}\right]$, the total Chern class of the isotropy representation is

$$
c(\iota \mathbb{C})=\prod_{i=1}^{4}\left(1-y_{i}^{2}\right) \prod_{\left(\epsilon_{1}, \epsilon_{2}, \epsilon_{3}\right) \in\{ \pm 1\}^{3}}\left(1-\frac{1}{4}\left(\epsilon_{1} y_{1}+\epsilon_{2} y_{2}+\epsilon_{3} y_{3}+y_{4}\right)^{2}\right) .
$$

\subsection{Isotropy representation for real forms of $F_{4}$}

$\boldsymbol{F}_{\mathbf{4}(4)}$ The maximal compact subgroup $K \subset F_{4(4)}$ has Lie algebra $\mathfrak{s u}_{2} \times \mathfrak{s p}_{3}$. As a $K$-representation, $\mathfrak{f}_{4}$ decomposes as

$$
\mathfrak{f}_{4}=\left(\mathfrak{s u}_{2} \oplus \mathfrak{s p}_{3}\right) \oplus \lambda_{2}^{1} \otimes W
$$

where $W \subset \lambda_{6}^{3}$ is the kernel of the contraction map $\lambda_{6}^{3} \rightarrow \lambda_{6}^{1}$ (it is an irreducible representation of $\mathrm{Sp}_{3}$ of dimension 14). 
Since $K \subset F_{4(4)}$ have the same rank, we can identify $\mathfrak{h}^{*}\left(\mathfrak{f}_{4}\right)=\left\langle J_{1}, J_{2}, J_{3}, J_{4}\right\rangle$ and $\mathfrak{h}^{*}\left(\mathfrak{s u}_{2} \times \mathfrak{s p}_{3}\right)=\left\langle L_{1}\right\rangle \oplus\left\langle L_{2}, L_{3}, L_{4}\right\rangle$.

The roots of $F_{4}$ are

$$
\begin{cases} \pm J_{i} & \text { if } 1 \leq i \leq 4 \\ \pm J_{i} \pm J_{j} & \text { if } 1 \leq i<j \leq 4 \\ \frac{1}{2}\left( \pm J_{1} \pm J_{2} \pm J_{3} \pm J_{4}\right) & \end{cases}
$$

According to [7, page 390], the roots of $\mathfrak{s u}_{2} \times \mathfrak{s p}_{3} \subset \mathfrak{f}_{4}$ are

$\pm\left(J_{1}-J_{2}\right), \quad \pm\left(J_{1}+J_{2}\right), \quad \pm J_{3}, \quad \pm J_{4}, \quad \pm J_{3} \pm J_{4}, \quad \frac{1}{2}\left( \pm\left(J_{1}+J_{2}\right) \pm J_{3} \pm J_{4}\right)$.

We can identify these roots with the roots of $\mathfrak{s u}_{2} \times \mathfrak{s p}_{3}$ inside $\mathfrak{h}^{*}\left(\mathfrak{s u}_{2} \times \mathfrak{s p}_{3}\right)$ by the identification

$$
\begin{aligned}
& J_{1}-J_{2} \longleftrightarrow 2 L_{1}, \\
& J_{1}+J_{2} \longleftrightarrow 2 L_{2}, \\
& J_{3}+J_{4} \longleftrightarrow 2 L_{3}, \\
& J_{3}-J_{4} \longleftrightarrow 2 L_{4} .
\end{aligned}
$$

Under this identification, the weights of the isotropy representation $\mathfrak{p}=W \otimes \lambda_{2}^{1}$ will be the roots of $\mathfrak{f}_{4}$ that are not roots of $\mathfrak{s u}_{2} \times \mathfrak{s p}_{3}$. Then the weights of the isotropy representation are $\pm L_{1} \pm L_{i}$ for $i=2,3,4$ and $\pm L_{1} \pm L_{2} \pm L_{3} \pm L_{4}$ (for all 16 sign choices).

Let $S \subset K$ be a maximal torus. As an element of $H^{*}(B S) \simeq \mathbb{Q}\left[y_{1}\right] \otimes \mathbb{Q}\left[y_{2}, y_{3}, y_{4}\right]$, the total Chern class for the isotropy representation is

(29) $c\left(\iota_{\mathbb{C}}\right)=\prod_{i=2}^{4}\left(1-\left(y_{1}+y_{i}\right)^{2}\right)\left(1-\left(y_{1}-y_{i}\right)^{2}\right) \prod\left(1-\left(y_{1}+\epsilon_{2} y_{2}+\epsilon_{3} y_{3}+\epsilon_{4} y_{4}\right)^{2}\right)$,

where the second product is over all tuples $\left(\epsilon_{2}, \epsilon_{3}, \epsilon_{4}\right) \in\{ \pm 1\}^{3}$.

$\boldsymbol{F}_{\mathbf{4}(-20)}$ The maximal compact subgroup $K \subset F_{4(-20)}$ has Lie algebra $\mathfrak{s o}_{9}$. Following [1, page 51], $\mathfrak{f}_{4}$ decomposes as a representation of $K$ as

$$
\mathfrak{f}_{4}=\mathfrak{s o}_{9} \oplus \Delta,
$$

where $\Delta$ is the spin representation of $K$. The isotropy representation $\mathfrak{p}=\Delta$ has weights $\frac{1}{2}\left( \pm L_{1} \pm \cdots \pm L_{4}\right)$.

Let $S \subset K$ be the maximal torus. As an element of $H^{*}(B S) \simeq \mathbb{Q}\left[y_{1}, \ldots, y_{4}\right]$, the total Chern class of the isotropy representation is

$$
c\left(\iota_{\mathbb{C}}\right)=\prod_{\left(\epsilon_{1}, \epsilon_{2}, \epsilon_{3}\right) \in\{ \pm 1\}^{3}}\left(1-\frac{1}{4}\left(\epsilon_{1} y_{1}+\epsilon_{2} y_{2}+\epsilon_{3} y_{3}+y_{4}\right)^{2}\right) .
$$




\subsection{Isotropy representation for real forms of $G_{2}$}

The maximal compact subgroup of $G_{2(2)}$ is $K=\mathrm{SU}_{2} \times \mathrm{SU}_{2}$. As a $K$-representation, $\mathfrak{g}_{2}$ decomposes as

$$
\mathfrak{g}_{2}=\left(\mathfrak{s u}_{2} \times \mathfrak{s u}_{2}\right)+\operatorname{Sym}^{2}(V) \otimes V,
$$

where $V$ is the standard representation of $\mathrm{SU}_{2}$, and $\operatorname{Sym}^{2}(V)$ is the $2^{\text {nd }}$ symmetric power. The weights of $\mathfrak{p}=\operatorname{Sym}^{2}(V) \otimes V$ are $\pm L_{1} \pm L_{2}$ and $\pm 3 L_{1} \pm L_{2}$. (Compare with [6, Lecture 22] or [7, page 393].)

Let $S \subset \mathrm{SU}_{2} \times \mathrm{SU}_{2}$ be a maximal torus. As an element of $H^{*}(B S) \simeq \mathbb{Q}\left[y_{1}, y_{2}\right]$, the total Chern class is

(31) $c\left(\iota_{\mathbb{C}}\right)=\left(1-\left(y_{2}+3 y_{1}\right)^{2}\right)\left(1-\left(y_{2}+y_{1}\right)^{2}\right)\left(1-\left(y_{2}-y_{1}\right)^{2}\right)\left(1-\left(y_{2}-3 y_{1}\right)^{2}\right)$.

\section{Computing $\alpha_{2}^{*}: H^{*}(B G) \rightarrow H^{*}\left(B G^{\delta}\right)$}

The following theorem allows us to compute $\alpha_{2}: H^{*}(B G) \rightarrow H^{*}\left(B G^{\delta}\right)$ for $G$ a real, semisimple Lie group.

Theorem 5.1 (Milnor [14, Theorem 2]) Let $G$ be a real, simple, connected Lie group. Assume that its complexification $G_{\mathbb{C}}$ is simple. Then the sequence

$$
H^{*}\left(B G_{\mathbb{C}} ; \mathbb{Q}\right) \stackrel{i^{*}}{\rightarrow} H^{*}(B G ; \mathbb{Q}) \stackrel{\alpha_{2}^{*}}{\longrightarrow} H^{*}\left(B G^{\delta} ; \mathbb{Q}\right)
$$

induced by the maps $G^{\delta} \rightarrow G \rightarrow G_{\mathbb{C}}$ is "exact" in the sense that the kernel of $\alpha_{2}^{*}$ is the ideal generated by the image of $i^{*}: H^{k}\left(B G_{\mathbb{C}}\right) \rightarrow H^{k}(B G)$ for $k>0$.

Theorem 5.1 applies to all the groups $G$ in Table 2. If $G$ is one of the complex Lie groups from Table 1, then $G_{\mathbb{C}} \simeq G \times G$ is not simple, so Theorem 5.1 does not apply. In this case, we have the following theorem, whose proof comes from Chern-Weil theory (see [14, Lemma 11]).

Theorem 5.2 Let $G$ be a complex, simple Lie group with finitely many components. Then $\alpha_{2}^{*}: H^{i}(B G ; \mathbb{Q}) \rightarrow H^{i}\left(B G^{\delta} ; \mathbb{Q}\right)$ is zero for $i>0$.

By Theorem 5.2, if $G$ is one of the groups from Table 1, then $\alpha_{2}^{*}$ is zero in positive degrees and so $p_{i}(\Gamma \backslash G / K)=0$ for $i>0$. This proves Theorem 1.2 for the groups in Table 1.

In the remainder of this section we use Theorem 5.1 to compute $\alpha_{2}^{*}$ for all the $G$ in Table 2. Let $G$ be one of the groups in Table 2 with maximal compact subgroup $K$. 
Let $G_{\mathbb{C}}$ be the complexification of $G$ and let $U$ be the maximal compact subgroup of $G_{\mathbb{C}}$. Let $S \subset K$ and $S^{\prime} \subset U$ be maximal tori such that complexification $G \rightarrow G_{\mathbb{C}}$ sends $S$ into $S^{\prime}$. We have the following diagram of inclusions, and an induced diagram on cohomology.
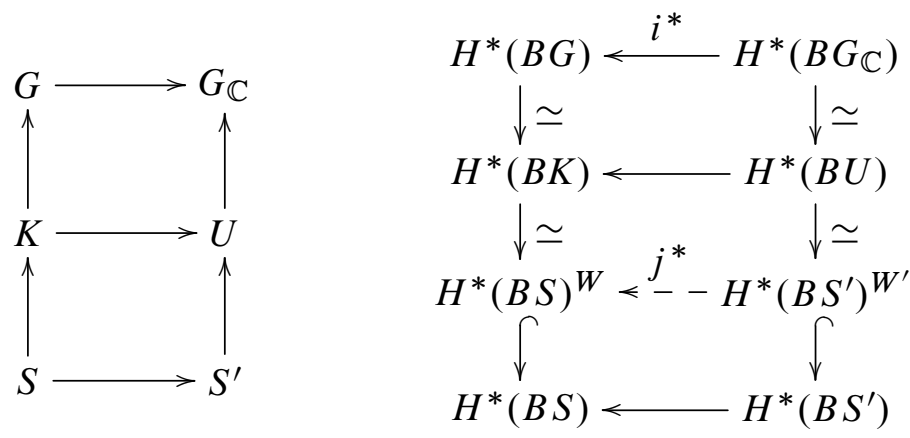

In the diagram on the right, the top two vertical arrows are isomorphisms because $K \hookrightarrow G$ and $U \hookrightarrow G_{\mathbb{C}}$ are homotopy equivalences. The middle two vertical arrows are isomorphisms by Theorem 3.1. Thus to compute the map $i^{*}$ it is enough to compute $j^{*}$. We do this in the remainder of this section.

\subsection{Computing $\alpha_{2}^{*}$ for $G=\mathrm{SL}_{n}(\mathbb{R})$}

Here $K=\mathrm{SO}_{n}$ and $U=\mathrm{SU}_{n}$. For $\theta \in \mathbb{R} / 2 \pi \mathbb{Z}$, let

$$
\underline{\theta}=\left(\begin{array}{rr}
\cos (\theta) & \sin (\theta) \\
-\sin (\theta) & \cos (\theta)
\end{array}\right)
$$

Let $k=[n / 2]$. The map $S \rightarrow S^{\prime}$ sends

$$
\left(\begin{array}{llll}
\underline{\theta}_{1} & & & \\
& \ddots & & \\
& & \underline{\theta}_{k} & \\
& & & (1)
\end{array}\right) \mapsto\left(\begin{array}{lll}
D & & \\
& D^{-1} & \\
& & \text { (1) }
\end{array}\right),
$$

where

$$
D=\left(\begin{array}{ccc}
e^{i \theta_{1}} & & \\
& \ddots & \\
& & e^{i \theta_{k}}
\end{array}\right),
$$

and the 1 in (32) appears only if $n$ is odd. Make the identifications

$$
H^{*}\left(B S^{\prime}\right)^{W^{\prime}} \simeq \frac{\operatorname{Sym}\left(x_{1} \ldots, x_{n}\right)}{\left(x_{1}+\cdots+x_{n}\right)}
$$


and

$$
H^{*}(B S)^{W} \simeq \begin{cases}\operatorname{Sym}\left(y_{1}^{2}, \ldots, y_{k}^{2}\right) & \text { if } n=2 k+1, \\ \left\langle\operatorname{Sym}\left(y_{1}^{2}, \ldots, y_{k}^{2}\right), y_{1} \cdots y_{k}\right\rangle & \text { if } n=2 k\end{cases}
$$

From (32) we see that the image of $j^{*}: H^{*}\left(B S^{\prime}\right)^{W^{\prime}} \rightarrow H^{*}(B S)^{W}$ is $\operatorname{Sym}\left(y_{1}^{2}, \ldots, y_{k}^{2}\right)$.

\subsection{Computing $\alpha_{2}^{*}$ for $G=\mathrm{SU}_{p, q}$}

Here $K=S\left(U_{p} \times U_{q}\right)$ and $U=\mathrm{SU}_{p+q}$, and the inclusion $S \hookrightarrow S^{\prime}$ is an isomorphism. Make the identifications

and

$$
H^{*}\left(B S^{\prime}\right)^{W^{\prime}} \simeq \frac{\operatorname{Sym}\left(x_{1} \ldots, x_{p+q}\right)}{\left(x_{1}+\cdots+x_{p+q}\right)}
$$

$$
H^{*}(B S)^{W} \simeq \frac{\operatorname{Sym}\left(y_{1} \ldots, y_{p}\right) \otimes \operatorname{Sym}\left(z_{1}, \ldots, z_{q}\right)}{\left(y_{1}+\cdots+y_{p}+z_{1}+\cdots+z_{q}\right)} .
$$

The image of $j^{*}: H^{*}\left(B S^{\prime}\right)^{W^{\prime}} \rightarrow H^{*}(B S)^{W}$ is $\operatorname{Sym}\left(y_{1}, \ldots, y_{p}, z_{1}, \ldots, z_{q}\right)$.

Corollary 5.3 Let $p, q \geq 2$ and let $G=\mathrm{SU}_{p, q}$. Then $\alpha_{2}^{*}: H^{*}(B G) \rightarrow H^{*}\left(B G^{\delta}\right)$ is injective on the linear subgroup generated by $\sum_{i<j} y_{i} y_{j}$ and $\left(\sum y_{i}\right)^{2}$.

Proof For $1 \leq i \leq p$ (resp. $1 \leq i \leq q)$, let $\sigma_{i}^{y}$ (resp. $\sigma_{i}^{z}$ ) denote the $i^{\text {th }}$ elementary symmetric polynomial in $\left\{y_{1}, \ldots, y_{p}\right\}$ (resp. $\left\{z_{1}, \ldots, z_{q}\right\}$ ), viewed as elements of $H^{*}(B S)^{W} \simeq H^{*}(B G)$. Similarly, let $\sigma_{i}^{y, z}$ denote the $i^{\text {th }}$ elementary symmetric polynomial in $\left\{y_{1}, \ldots, y_{p}, z_{1}, \ldots, z_{q}\right\}$. Let $\mathcal{I}$ denote the ideal

$$
\mathcal{I}=\left(\sigma_{1}^{y, z}, \ldots, \sigma_{p+q}^{y, z}\right) .
$$

The following relations are easy to verify:

$$
\sigma_{1}^{y, z}=\sigma_{1}^{y}+\sigma_{1}^{z} \quad \text { and } \quad \sigma_{2}^{y, z}=\sigma_{2}^{y}+\sigma_{1}^{y} \sigma_{1}^{z}+\sigma_{2}^{z} .
$$

Note that $\left(\sigma_{1}^{y}\right)^{2}=\left(\sum y_{i}\right)^{2}$ and $\sigma_{2}^{y}=\sum_{i<j} y_{i} y_{j}$. By Theorem 5.1, to prove the corollary it is enough to show that no nontrivial linear combination $c \cdot \sigma_{2}^{y}+d \cdot\left(\sigma_{1}^{y}\right)^{2}$ belongs to $\mathcal{I}$. The elements of $\mathcal{I}$ that have total degree 2 all have the form

$$
\left(a \sigma_{1}^{y}+b \sigma_{1}^{z}\right) \cdot \sigma_{1}^{y, z}+m \cdot \sigma_{2}^{y, z},
$$

where $a, b, m$ are scalars. By simple linear algebra, one checks that if

$$
c \cdot \sigma_{2}^{y}+d \cdot\left(\sigma_{1}^{y}\right)^{2}=\left(a \sigma_{1}^{y}+b \sigma_{1}^{z}\right) \cdot \sigma_{1}^{y, z}+m \cdot \sigma_{2}^{y, z},
$$

then $m=a=b=0$. This implies $c=d=0$ because $\sigma_{2}^{y}$ and $\left(\sigma_{1}^{y}\right)^{2}$ are linearly independent in $H^{*}(B S)^{W}$. Hence no nontrivial linear combination $c \cdot \sigma_{2}^{y}+d \cdot\left(\sigma_{2}^{y}\right)^{2}$ belongs to $\mathcal{I}$. 
Corollary 5.4 Let $p \geq 2$ and let $G=\mathrm{SU}_{p, 1}$. Then $\alpha_{2}^{*}: H^{*}(B G) \rightarrow H^{*}\left(B G^{\delta}\right)$ is nonzero on $\sum_{i<j} y_{i} y_{j}$, and

$$
\alpha_{2}^{*}\left(\sum y_{i}\right)^{2}=\alpha_{2}^{*}\left(\sum_{i<j} y_{i} y_{j}\right) .
$$

Proof We use the same notation as in the proof of Corollary 5.3. Since $q=1$ in the present case, the relations in (33) simplify to the following relations:

$$
\sigma_{1}^{y, z}=\sigma_{1}^{y}+\sigma_{1}^{z} \quad \text { and } \quad \sigma_{2}^{y, z}=\sigma_{2}^{y}+\sigma_{1}^{y} \sigma_{1}^{z} .
$$

Since the elements $\sigma_{i}^{y}, \sigma_{j}^{z}$ are all linearly independent in $H^{2}(B S)^{W}$, it is a simple matter of linear algebra to show that $\sigma_{2}^{y}$ is not in $\mathcal{I}$, and hence is not in the kernel of $\alpha_{2}^{*}$.

To see that $\alpha_{2}^{*}\left(\left(\sigma_{1}^{y}\right)^{2}\right)=\alpha_{2}^{*}\left(\sigma_{2}^{y}\right)$, note that the first equation in (34) implies that

$$
\sigma_{1}^{y} \cdot \sigma_{1}^{y, z}=\left(\sigma_{1}^{y}\right)^{2}+\sigma_{1}^{y} \sigma_{1}^{z} \in \mathcal{I} \text {. }
$$

Then $\sigma_{2}^{y}-\left(\sigma_{1}^{y}\right)^{2}=\sigma_{2}^{y, z}-\sigma_{1}^{y} \cdot \sigma_{1}^{y, z}$ belongs to $\mathcal{I}$ (the right-hand side obviously does). This implies that $\sigma_{2}^{y}$ and $\left(\sigma_{1}^{y}\right)^{2}$ have the same image under $\alpha_{2}^{*}$.

\subsection{Computing $\alpha_{2}^{*}$ for $G=\operatorname{Sp}_{2 n}(\mathbb{R})$}

Here $K=U_{n}$ and $U=\operatorname{Sp}(n)$, and the inclusion $S \hookrightarrow S^{\prime}$ is an isomorphism. Make the identifications

$$
H^{*}\left(B S^{\prime}\right)^{W^{\prime}} \simeq \operatorname{Sym}\left(x_{1}^{2}, \ldots, x_{n}^{2}\right) \text { and } H^{*}(B S)^{W} \simeq \operatorname{Sym}\left(y_{1}, \ldots, y_{n}\right) .
$$

The image of $j^{*}: H^{*}\left(B S^{\prime}\right)^{W^{\prime}} \rightarrow H^{*}(B S)^{W}$ is $\operatorname{Sym}\left(y_{1}^{2}, \ldots, y_{n}^{2}\right)$.

Corollary 5.5 Let $G=\operatorname{Sp}_{2 n}(\mathbb{R})$. Then $\alpha_{2}^{*}\left(\sum y_{i}\right)^{2} \neq 0$.

Proof Let $\sigma_{i}$ (resp. $\left.\omega_{i}\right)$ be the $i^{\text {th }}$ elementary symmetric polynomial in $\left\{y_{1}, \ldots, y_{n}\right\}$ (resp. $\left.\left\{y_{1}^{2}, \ldots, y_{n}^{2}\right\}\right)$. Let $\mathcal{I}$ denote the ideal $\left(\omega_{1}, \ldots, \omega_{n}\right)$.

By Theorem 5.1, to show $\sigma_{1}^{2}=\left(\sum y_{i}\right)^{2}$ is not in $\operatorname{ker} \alpha_{2}^{*}$, it is enough to show $\sigma_{1}^{2} \notin \mathcal{I}$. Note that $\omega_{1}=\sigma_{1}^{2}-2 \sigma_{2}$ and that the only elements of $\mathcal{I}$ of degree 2 are scalar multiples of $\omega_{1}$. Since $\sigma_{1}^{2}$ is obviously not a multiple of $\sigma_{1}^{2}-2 \sigma_{2}$, we conclude $\sigma_{1}^{2} \notin \mathcal{I}$. 


\subsection{Computing $\alpha_{2}^{*}$ for $G=\mathrm{SO}_{p, q}$}

Here $K=\mathrm{SO}_{p} \times \mathrm{SO}_{q}$ and $U=\mathrm{SO}_{p+q}$. Let $m=[(p+q) / 2]$, let $a=[p / 2]$, and let $b=[q / 2]$. Make the identifications

$$
H^{*}\left(B S^{\prime}\right) \simeq \mathbb{Q}\left[x_{1}, \ldots, x_{m}\right] \text { and } H^{*}(B S) \simeq\left[y_{1}, \ldots, y_{p}, z_{1}, \ldots, z_{q}\right] .
$$

It is not hard to see that the image of $j^{*}: H^{*}\left(B S^{\prime}\right)^{W^{\prime}} \rightarrow H^{*}(B S)^{W}$ contains $\operatorname{Sym}\left(y_{1}^{2}, \ldots, y_{p}^{2}, z_{1}^{2}, \ldots, z_{q}^{2}\right)$.

Corollary 5.6 Let $p, q \geq 2$ and let $G=\mathrm{SO}_{p, q}$. Then $\alpha_{2}^{*}\left(\sum y_{i}^{2}\right) \neq 0$.

Proof The proof is the same as in Corollary 5.5. The image of

$$
H^{4}\left(B S^{\prime}\right)^{W^{\prime}} \simeq H^{4}\left(B G_{\mathbb{C}}\right) \rightarrow H^{4}(B G) \simeq H^{4}(B S)^{W}
$$

is generated by multiples of $\sum y_{i}^{2}+\sum z_{j}^{2}$, and $\sum y_{i}^{2}$ does not have this form.

Corollary 5.7 Let $p \geq 4$ and let $G=\mathrm{SO}_{p, p}$. Then $\alpha_{2}^{*}: H^{*}(B G) \rightarrow H^{*}\left(B G^{\delta}\right)$ is injective on the linear subgroup generated by $\sum_{i<j} y_{i}^{2} y_{j}^{2}$ and $\left(\sum y_{i}^{2}\right)^{2}$.

Proof The proof is identical to the proof of Corollary 5.3 after replacing $y_{i}$ and $z_{j}$ by $y_{i}^{2}$ and $z_{j}^{2}$.

\subsection{Computing $\alpha_{2}^{*}$ for $G=\mathrm{Sp}_{p, q}$}

Here $K=\operatorname{Sp}(p) \times \operatorname{Sp}(q)$ and $U=\operatorname{Sp}(p+q)$, and the inclusion $S \hookrightarrow S^{\prime}$ is an isomorphism. Make the identifications

$$
H^{*}\left(B S^{\prime}\right)^{W^{\prime}} \simeq \operatorname{Sym}\left(x_{1}^{2}, \ldots, x_{p+q}^{2}\right)
$$

and

$$
H^{*}(B S)^{W} \simeq \operatorname{Sym}\left(y_{1}^{2}, \ldots, y_{p}^{2}\right) \otimes \operatorname{Sym}\left(z_{1}^{2}, \ldots, z_{q}^{2}\right) .
$$

The image of $j^{*}: H^{*}\left(B S^{\prime}\right)^{W^{\prime}} \rightarrow H^{*}(B S)^{W}$ is $\operatorname{Sym}\left(y_{1}^{2}, \ldots, y_{p}^{2}, z_{1}^{2}, \ldots, z_{q}^{2}\right)$. We have Corollaries 5.8 and 5.9, whose proofs are identical to the proofs for Corollaries 5.6 and 5.7 , respectively.

Corollary 5.8 Let $p, q \geq 1$ and let $G=\operatorname{Sp}_{p, q}$. Then $\alpha_{2}^{*}\left(\sum y_{i}^{2}\right) \neq 0$.

Corollary 5.9 Let $q \geq 1$ and $p \geq 2$, and let $G=\operatorname{Sp}_{p, q}$. Then $\alpha_{2}^{2}$ is injective on $\left(\sum y_{i}\right)^{2}$. If $p, q \geq 2$, then $\alpha_{2}^{*}$ is injective on the linear subgroup generated by $\sum_{i<j} y_{i}^{2} y_{j}^{2}$ and $\left(\sum y_{i}^{2}\right)^{2}$. 


\subsection{Computing $\alpha_{2}^{*}$ for $G=\mathrm{SO}_{2 n}^{*}$}

Here $K=U_{n}$ and $U=\mathrm{SO}_{2 n}$, and the inclusion $S \hookrightarrow S^{\prime}$ is an isomorphism. Make the identifications

$$
H^{*}\left(B S^{\prime}\right) \simeq \mathbb{Q}\left[x_{1}, \ldots, x_{n}\right] \text { and } H^{*}(B S) \simeq \mathbb{Q}\left[y_{1}, \ldots, y_{n}\right] .
$$

The image of

$$
j^{*}: H^{*}\left(B S^{\prime}\right)^{W^{\prime}} \rightarrow H^{*}(B S)^{W}=\operatorname{Sym}\left(y_{1}, \ldots, y_{n}\right)
$$

contains $\operatorname{Sym}\left(y_{1}^{2}, \ldots, y_{n}^{2}\right)$. We have the following corollary, whose proof is identical to the proof of Corollary 5.5.

Corollary 5.10 Let $G=\mathrm{SO}_{2 n}^{*}$. Then $\alpha_{2}^{*}\left(\sum y_{i}\right)^{2} \neq 0$.

\subsection{Computing $\alpha_{2}^{*}$ for $G=\mathrm{SU}_{2 n}^{*}$}

Here $K=\mathrm{Sp}_{n}$ and $U=\mathrm{SU}_{2 n}$. Make the identifications

and

$$
H^{*}\left(B S^{\prime}\right)^{W^{\prime}} \simeq \frac{\operatorname{Sym}\left(x_{1}, \ldots, x_{2 n}\right)}{\left(x_{1}+\cdots+x_{2 n}\right)}
$$

$$
H^{*}(B S)^{W} \simeq \operatorname{Sym}\left(y_{1}^{2}, \ldots, y_{n}^{2}\right) .
$$

It is not hard to see that $j^{*}: H^{*}\left(B S^{\prime}\right)^{W^{\prime}} \rightarrow H^{*}(B S)^{W}$ is surjective.

Corollary 5.11 Let $G=\mathrm{SU}_{2 n}^{*}$. For $i>0$, the map $\alpha_{2}^{*}: H^{i}(B G) \rightarrow H^{i}\left(B G^{\delta}\right)$ is zero.

\subsection{Computing $\alpha_{2}^{*}$ for $G$ a real form of $E_{8}$}

$\boldsymbol{E}_{\mathbf{8}(\mathbf{8})} \operatorname{Here} \operatorname{Lie}(K)=\mathfrak{s o}_{16}$ and $U=E_{8}$, and the inclusion $S \hookrightarrow S^{\prime}$ is an isomorphism. As in Section 3, we identify

$$
H^{1}\left(S^{\prime}\right) \simeq\left\langle J_{1}, \ldots, J_{8}\right\rangle \text { and } H^{1}(S) \simeq\left\langle L_{1}, \ldots, L_{8}\right\rangle .
$$

Under $H^{1}\left(S^{\prime}\right) \rightarrow H^{1}(S)$ we have $J_{i} \mapsto L_{i}$. Let $z_{i}=\tau\left(J_{i}\right) \in H^{2}\left(B S^{\prime}\right)$ and let $y_{i}=\tau\left(L_{i}\right) \in H^{2}(B S)$. In Section 3, we explained that

$$
H^{*}\left(B S^{\prime}\right)^{W^{\prime}} \simeq \mathbb{Q}\left[I_{2}, I_{8}, I_{12}, I_{14}, I_{18}, I_{20}, I_{24}, I_{30}\right]
$$


Under $j^{*}: H^{4}\left(B S^{\prime}\right) \rightarrow H^{4}(B S)$ the polynomial $I_{2}$ maps to a multiple of $y_{1}^{2}+\cdots+y_{8}^{2}$. By Theorem 5.1 the elements of $H^{8}(B S)^{W}$ in the kernel $\alpha_{2}^{*}$ are multiples of

$$
\left(y_{1}^{2}+\cdots+y_{8}^{2}\right)^{2}=\left(\sum_{i=1}^{8} y_{i}^{4}\right)+2\left(\sum_{1 \leq i<j \leq 8} y_{i}^{2} y_{j}^{2}\right) .
$$

This implies the following corollary:

Corollary 5.12 Let $G=E_{8(8)}$. With the notation above, $\alpha_{2}^{*}\left(\sum_{i=1}^{8} y_{i}^{4}\right) \neq 0$.

$\boldsymbol{E}_{\mathbf{8}(-24)}$ Here $K=E_{7} \times \mathrm{SU}_{2}$ and $U=E_{8}$, and the inclusion $S \hookrightarrow S^{\prime}$ is an isomorphism. Make the identifications $H^{1}\left(S^{\prime}\right) \simeq\left\langle J_{1}, \ldots, J_{8}\right\rangle$ and

$$
H^{1}(S) \simeq\left\langle J_{1}, \ldots, J_{6}, J_{7}+J_{8}\right\rangle \oplus\left\langle J_{7}-J_{8}\right\rangle .
$$

Let $z_{i}=\tau\left(L_{i}\right) \in H^{2}\left(B S^{\prime}\right)$ for $1 \leq i \leq 8$. Let $y_{i}=\tau\left(L_{i}\right) \in H^{2}(B S)$ for $1 \leq i \leq 6$, $y_{7}=\tau\left(J_{7}+J_{8}\right)$ and $y_{8}=\tau\left(L_{7}-L_{8}\right)$. In Section 3 we explained that

$$
H^{*}\left(B S^{\prime}\right)^{W^{\prime}} \simeq \mathbb{Q}\left[I_{2}, I_{8}, I_{12}, I_{14}, I_{18}, I_{20}, I_{24}, I_{30}\right],
$$

and $I_{2}=30\left(z_{1}^{2}+\cdots+z_{8}^{2}\right)$. Under $j^{*}: H^{2}\left(B S^{\prime}\right) \rightarrow H^{2}(B S), z_{i} \mapsto y_{i}$ for $1 \leq i \leq 6$, $z_{7} \mapsto \frac{1}{2}\left(y_{7}+y_{8}\right)$ and $z_{8} \mapsto \frac{1}{2}\left(y_{7}-y_{8}\right)$, so

$$
j^{*}\left(I_{2}\right)=30\left(y_{1}^{2}+\cdots+y_{6}^{2}\right)+15\left(y_{7}^{2}+y_{8}^{2}\right) .
$$

By Theorem 5.1, every element of the kernel $H^{4}\left(B E_{8(-24)}\right) \rightarrow H^{4}\left(B\left(E_{8(-24)}\right)^{\delta}\right)$ is a scalar multiple of $j^{*}\left(I_{2}\right)$. This implies the following corollary:

Corollary 5.13 Let $G=E_{8(-24)}$. With the notation above, $\alpha_{2}^{*}\left(y_{8}^{2}\right) \neq 0$.

\subsection{Computing $\alpha_{2}^{*}$ for $G$ a real form of $E_{7}$}

$\boldsymbol{E}_{\mathbf{7}(7)}$ Here $\operatorname{Lie}(K)=\mathfrak{s u}_{8}$ and $U=E_{7}$, and the inclusion $S \hookrightarrow S^{\prime}$ is an isomorphism. We identify $H^{1}\left(S^{\prime}\right)$ as the subspace of $\left\langle J_{1}, \ldots, J_{8}\right\rangle$ orthogonal to $J_{1}+\cdots+J_{8}$, and we identify $H^{1}(S)$ as the subspace of $\left\langle L_{1}, \ldots, L_{8}\right\rangle$ orthogonal to $L_{1}+\cdots+L_{8}$. Then $H^{1}\left(S^{\prime}\right) \rightarrow H^{1}(S)$ is the obvious map $J_{i} \mapsto L_{i}$. Let $z_{i}=\tau\left(J_{i}\right) \in H^{2}\left(B S^{\prime}\right)$ and $y_{i}=\tau\left(L_{i}\right) \in H^{2}(B S)$. In Section 3 we explained that

$$
H^{*}\left(B S^{\prime}\right)^{W^{\prime}} \simeq \mathbb{Q}\left[I_{2}, I_{6}, I_{8}, I_{10}, I_{12}, I_{14}, I_{18}\right],
$$

and computed $I_{2}=\frac{3}{4}\left[7\left(\sum z_{i}^{2}\right)+2\left(\sum z_{i} z_{j}\right)\right]$ for this copy of $E_{7} \subset E_{8}$. Under $j^{*}: H^{*}\left(B S^{\prime}\right) \rightarrow H^{*}(B S)$,

$$
I_{2} \mapsto \frac{3}{4}\left[7\left(\sum y_{i}^{2}\right)+2\left(\sum y_{i} y_{j}\right)\right]=-9\left(\sum y_{i} y_{j}\right) .
$$


The equality follows because of the relation $\sum y_{i}^{2}=-2 \sum y_{i} y_{j}$ in $H^{*}(B S)^{W}$. By Theorem 5.1, every element of the kernel of

$$
\alpha_{2}^{*}: H^{8}\left(B E_{7(7)}\right) \rightarrow H^{8}\left(B\left(E_{7(7)}\right)^{\delta}\right)
$$

is a scalar multiple of $\left(\sum y_{i}^{2}\right)^{2}$. This implies the following corollary:

Corollary 5.14 Let $G=E_{7(7)}$. With the notation above, $\alpha_{2}^{*}\left(\sum y_{i}^{4}\right) \neq 0$.

$\boldsymbol{E}_{\mathbf{7}(-\mathbf{5})}$ Here $\operatorname{Lie}(K)=\mathfrak{s o}_{12} \times \mathfrak{s u}_{2}$ and $U=E_{7}$, and the inclusion $S \hookrightarrow S^{\prime}$ is an isomorphism. As in Section 3, we identify

$$
H^{1}\left(S^{\prime}\right) \simeq\left\langle J_{1}, \ldots, J_{6}, J_{7}+J_{8}\right\rangle \text { and } H^{1}(S) \simeq\left\langle L_{1}, \ldots, L_{6}\right\rangle \oplus\left\langle L_{7}\right\rangle .
$$

Under $H^{1}\left(S^{\prime}\right) \rightarrow H^{1}(S)$, we have

$$
\left\{\begin{aligned}
J_{i} & \mapsto L_{i} \\
J_{7}+J_{8} & \mapsto 2 L_{7}
\end{aligned} \quad \text { if } 1 \leq i \leq 6,\right.
$$

Let $z_{i}=\tau\left(J_{i}\right) \in H^{2}\left(B S^{\prime}\right)$ for $1 \leq i \leq 6$ and let $z_{7}=\tau\left(J_{7}+J_{8}\right)$. Let $y_{i}=$ $\tau\left(L_{i}\right) \in H^{2}(B S)$. In Section 3 we explained that

$$
H^{*}\left(B S^{\prime}\right)^{W^{\prime}} \simeq \mathbb{Q}\left[I_{2}, I_{6}, I_{8}, I_{10}, I_{12}, I_{14}, I_{18}\right] .
$$

Under $j^{*}: H^{4}\left(B S^{\prime}\right) \rightarrow H^{4}(B S)$,

$$
I_{2} \mapsto 6\left(y_{1}^{2}+y_{2}^{2}+y_{3}^{2}+y_{4}^{2}+y_{5}^{2}+y_{6}^{2}+2 y_{7}^{2}\right) .
$$

In particular, by Theorem 5.1 every element of the kernel of

$$
\alpha_{2}^{*}: H^{4}\left(B E_{7(-5)}\right) \rightarrow H^{4}\left(B\left(E_{7(-5)}\right)^{\delta}\right)
$$

is a scalar multiple of $j^{*}\left(I_{2}\right)$. This implies the following corollary:

Corollary 5.15 Let $G=E_{7(5)}$. With the notation above, $\alpha_{2}^{*}\left(y_{7}^{2}\right) \neq 0$.

$\boldsymbol{E}_{\mathbf{7 ( - 2 5 )}}$ Here $\operatorname{Lie}(K)=\mathfrak{e}_{6} \times \mathfrak{s o}_{2}$ and $U=E_{7}$, and the inclusion $S \hookrightarrow S^{\prime}$ is an isomorphism. Make the identifications $H^{1}\left(S^{\prime}\right) \simeq\left\langle J_{1}, \ldots, J_{6}, J_{7}+J_{8}\right\rangle$ and

$$
H^{1}(S) \simeq\left\langle J_{1}, \ldots, J_{5}, J_{6}+J_{7}+J_{8}\right\rangle \oplus\left\langle 2 J_{6}-J_{7}-J_{8}\right\rangle .
$$

Let $z_{i}=\tau\left(J_{i}\right) \in H^{2}\left(B S^{\prime}\right)$ for $1 \leq i \leq 6$, and let $z_{7}=\tau\left(J_{7}+J_{8}\right)$. For $1 \leq i \leq 5$, let $y_{i}=\tau\left(J_{i}\right) \in H^{2}(B S)$, and let $y_{6}=\tau\left(J_{6}+J_{7}+J_{8}\right)$ and $y_{7}=\tau\left(2 J_{6}-J_{7}-J_{8}\right)$.

In Section 3 we explained that

$$
H^{*}\left(B S^{\prime}\right)^{W^{\prime}} \simeq \mathbb{Q}\left[I_{2}, I_{6}, I_{8}, I_{10}, I_{12}, I_{14}, I_{18}\right]
$$


where $I_{2}=6\left(z_{1}^{2}+\cdots+z_{6}^{2}\right)+3 z_{7}^{2}$. Under $j^{*}: H^{2}\left(B S^{\prime}\right) \rightarrow H^{2}(B S), z_{i} \mapsto y_{i}$ for $1 \leq i \leq 5, z_{6} \mapsto \frac{1}{3}\left(y_{6}-y_{7}\right)$ and $z_{7} \mapsto \frac{1}{3}\left(2 y_{6}+y_{7}\right)$, so

$$
j^{*}\left(I_{2}\right)=6\left(y_{1}^{2}+y_{2}^{2}+y_{3}^{2}+y_{4}^{2}+y_{5}^{2}\right)+2 y_{6}^{2}+y_{7}^{2} .
$$

By Theorem 5.1, $j^{*}\left(I_{2}\right)$ generates the kernel of

$$
\alpha_{2}^{*}: H^{4}\left(B E_{7(-25)}\right) \rightarrow H^{4}\left(B\left(E_{7(-25)}\right)^{\delta}\right) .
$$

In particular, we have the following corollary:

Corollary 5.16 Let $G=E_{7(-25)}$. With the notation above, $\alpha_{2}^{*}\left(y_{7}^{2}\right) \neq 0$.

\subsection{Computing $\alpha_{2}^{*}$ for $G$ a real form of $E_{6}$}

$\boldsymbol{E}_{\mathbf{6}(6)}$ Here $\operatorname{Lie}(K)=\mathfrak{s p}_{4}$ and $U=E_{6}$. Make the identifications $H^{1}(S) \simeq$ $\left\langle L_{1}, \ldots, L_{4}\right\rangle$ and

$$
H^{1}\left(S^{\prime}\right) \subset\left\langle J_{1}, \ldots, J_{6}, J_{7}-J_{8}\right\rangle
$$

as the subspace orthogonal to the roots in (7). Under $\mathfrak{s p}_{4} \hookrightarrow \mathfrak{e}_{6} \hookrightarrow \mathfrak{e}_{8}$ the Cartan subalgebra $\mathfrak{h}\left(\mathfrak{s p}_{4}\right) \subset \mathfrak{s p}_{4}$ is contained in the image of the Cartan subalgebra for $\mathfrak{s u}_{6} \times \mathfrak{s u}_{2} \subset \mathfrak{s u}_{8}$. Under $\mathfrak{h}^{*}\left(\mathfrak{e}_{8}\right) \rightarrow \mathfrak{h}^{*}\left(\mathfrak{s p}_{4}\right)$, we have

$$
\left\{\begin{array}{rlrl}
J_{i} & \mapsto L_{i} & \text { if } 1 \leq i \leq 3, \\
J_{i+3} & \mapsto-L_{i} & \text { if } 1 \leq i \leq 3, \\
J_{7} & \mapsto L_{4}, & & \\
J_{8} & \mapsto-L_{4} . &
\end{array}\right.
$$

It is then easy to determine the restriction of this map to $\mathfrak{h}^{*}\left(\mathfrak{e}_{6}\right)$.

Let $z_{i}=\tau\left(J_{i}\right)$ for $1 \leq i \leq 6$ and $z_{7}=\tau\left(J_{7}-J_{8}\right)$ in $H^{2}\left(B S^{\prime}\right)$. Let $y_{i}=$ $\tau\left(L_{i}\right) \in H^{2}(B S)$ for $1 \leq i \leq 4$. By Section $3, H^{*}\left(B S^{\prime}\right)^{W^{\prime}} \simeq \mathbb{Q}\left[I_{2}, I_{5}, I_{6}, I_{8}, I_{9}, I_{12}\right]$, where

$$
I_{2}=5\left(z_{1}^{2}+\cdots+z_{6}^{2}\right)+3 z_{7}^{2}-2 \sum_{1 \leq i<j \leq 6} z_{i} z_{j} .
$$

Under $j^{*}: H^{*}\left(B S^{\prime}\right) \rightarrow H^{*}(B S)$,

$$
I_{2} \mapsto 12\left(y_{1}^{2}+y_{2}^{2}+y_{3}^{2}+y_{4}^{2}\right) .
$$

By Theorem 5.1, $j^{*}\left(I_{2}\right)$ and $j^{*}\left(I_{2}\right)^{2}$ generate (as a vector space) the kernel of

$$
\alpha_{2}^{*}: H^{k}\left(B E_{6(6)}\right) \rightarrow H^{k}\left(B\left(E_{6(6)}\right)^{\delta}\right)
$$

for $k=4,8$. In particular, we have the following corollary: 
Corollary 5.17 Let $G=E_{6(6)}$. With the notation above, $\alpha_{2}^{*}\left(\sum y_{i}^{4}\right) \neq 0$.

$\boldsymbol{E}_{\mathbf{6}(2)}$ Here $\operatorname{Lie}(K)=\mathfrak{s u}_{6} \times \mathfrak{s u}_{2}$ and $U=E_{6}$, and the inclusion $S \hookrightarrow S^{\prime}$ is an isomorphism. We identify $H^{1}\left(S^{\prime}\right) \subset\left\langle J_{1}, \ldots, J_{6}, J_{7}-J_{8}\right\rangle$ as the subspace of $\left\langle J_{1}, \ldots, J_{8}\right\rangle$ orthogonal to the roots in (7). We identify $H^{1}(S)$ as the subspace of $\left\langle L_{1}, \ldots, L_{6}\right\rangle \oplus\left\langle L_{7}\right\rangle$ orthogonal to $L_{1}+\cdots+L_{6}$. The map $H^{1}\left(S^{\prime}\right) \rightarrow H^{1}(S)$ is given by $J_{i} \mapsto L_{i}$ for $1 \leq i \leq 6$ and $J_{7}-J_{8} \mapsto 2 L_{i}$.

Let $z_{i}=\tau\left(J_{i}\right) \in H^{2}\left(B S^{\prime}\right)$ for $1 \leq i \leq 6$ and let $z_{7}=\tau\left(J_{7}-J_{8}\right)$. Let $y_{i}=$ $\tau\left(L_{i}\right) \in H^{2}(B S)$ for $1 \leq i \leq 7$. In Section 3, we explained that

$$
H^{*}\left(B S^{\prime}\right)^{W^{\prime}} \simeq \mathbb{Q}\left[I_{2}, I_{5}, I_{6}, I_{8}, I_{9}, I_{12}\right]
$$

where

$$
I_{2}=5\left(z_{1}^{2}+\cdots+z_{6}^{2}\right)+3 z_{7}^{2}-2 \sum_{1 \leq i<j \leq 6} z_{i} z_{j} .
$$

Under $j^{*}: H^{*}\left(B S^{\prime}\right) \rightarrow H^{*}(B S)$,

$$
I_{2} \mapsto 5\left(y_{1}^{2}+\cdots+y_{6}^{2}\right)+12 y_{7}^{2}-2 \sum_{1 \leq i<j \leq 6} y_{i} y_{j} .
$$

Since $\sum y_{i}^{2}=-2 \sum y_{i} y_{j}$ in $H^{*}\left(B \mathrm{SU}_{6}\right)$, we can re-write this polynomial as

$$
j^{*}\left(I_{2}\right)=6\left(y_{1}^{2}+\cdots+y_{6}^{2}+2 y_{7}^{2}\right) .
$$

By Theorem 5.1, the kernel of

$$
\alpha_{2}^{*}: H^{4}\left(B E_{6(2)}\right) \rightarrow H^{4}\left(B\left(E_{6(2)}\right)^{\delta}\right)
$$

is generated by scalar multiples of $j^{*}\left(I_{2}\right)$. In particular, we have the following corollary:

Corollary 5.18 Let $G=E_{6(2)}$. With the notation above, $\alpha_{2}^{*}\left(y_{7}^{2}\right) \neq 0$.

$\boldsymbol{E}_{\mathbf{6}(-14)}$ Here $\operatorname{Lie}(K)=\mathfrak{s o}_{10} \times \mathfrak{s o}_{2}$ and $U=E_{6}$, and the inclusion $S \hookrightarrow S^{\prime}$ is an isomorphism. As in Section 3 we identify

$$
H^{1}\left(S^{\prime}\right) \simeq\left\langle J_{1}, \ldots, J_{5}, J_{6}+J_{7}+J_{8}\right\rangle \quad \text { and } \quad H^{1}(S) \simeq\left\langle L_{1}, \ldots, L_{5}\right\rangle \oplus\left\langle L_{6}\right\rangle .
$$

Under $H^{1}\left(S^{\prime}\right) \rightarrow H^{1}(S)$ we have $J_{i} \mapsto L_{i}$ for $1 \leq i \leq 5$ and $J_{6}+J_{7}+J_{8} \mapsto L_{6}$. Let $z_{i}=\tau\left(J_{i}\right) \in H^{2}\left(B S^{\prime}\right)$ for $1 \leq i \leq 5$ and let $w=\tau\left(J_{6}+J_{7}+J_{8}\right)$. Let $y_{i}=\tau\left(L_{i}\right) \in H^{2}(B S)$. In Section 3, we explained that

$$
H^{*}\left(B S^{\prime}\right)^{W^{\prime}} \simeq \mathbb{Q}\left[I_{2}, I_{5}, I_{6}, I_{8}, I_{9}, I_{12}\right] .
$$


Under $H^{4}\left(B S^{\prime}\right) \rightarrow H^{4}(B S)$,

$$
I_{2} \mapsto 6\left(y_{1}^{2}+y_{2}^{2}+y_{3}^{2}+y_{4}^{2}+y_{5}^{2}\right)+3 y_{6}^{2} .
$$

In particular, by Theorem 5.1 every element of the kernel of

$$
\alpha_{2}^{*}: H^{4}\left(B E_{6(-14)}\right) \rightarrow H^{4}\left(B\left(E_{6(-14)}\right)^{\delta}\right)
$$

is a scalar multiple of $j^{*}\left(I_{2}\right)$. This implies the following corollary:

Corollary 5.19 Let $G=E_{6(-14)}$. With the notation above, $\alpha_{2}^{*}\left(y_{6}^{2}\right) \neq 0$.

$\boldsymbol{E}_{\mathbf{6}(-26)}$ Here $K=F_{4}$ and $U=E_{6}$. Make the identifications

$$
H^{1}(S) \simeq\left\langle L_{1}, \ldots, L_{4}\right\rangle \quad \text { and } \quad H^{1}\left(S^{\prime}\right)=\left\langle J_{1}, \ldots, J_{5}, J_{6}+J_{7}+J_{8}\right\rangle .
$$

Let $z_{i}=\tau\left(J_{i}\right) \in H^{2}\left(B S^{\prime}\right)$ for $1 \leq i \leq 5$ and let $z_{6}=\tau\left(J_{6}+J_{7}+J_{8}\right)$. For $1 \leq i \leq 4$, let $y_{i}=\tau\left(L_{i}\right) \in H^{2}(B S)$.

Under $H^{*}\left(B S^{\prime}\right) \rightarrow H^{*}(B S)$ we have $z_{5}, z_{6} \mapsto 0$ and $z_{i} \mapsto y_{i}$ for $i=1, \ldots, 4$. By (8) and (6), it is easy to see that $j^{*}$ is surjective.

Corollary 5.20 Let $G=E_{6(-26)}$. For $i>0$, the map $\alpha_{2}^{*}: H^{i}(B G) \rightarrow H^{i}\left(B G^{\delta}\right)$ is zero.

\subsection{Computing $\alpha_{2}^{*}$ for $G$ a real form of $F_{4}$}

$\boldsymbol{F}_{4(4)} \quad$ Here $\operatorname{Lie}(K)=\mathfrak{s u}_{2} \times \mathfrak{s p}_{3}$ and $U=F_{4}$, and the inclusion $S \hookrightarrow S^{\prime}$ is an isomorphism. As in Section 3 we identify $H^{1}\left(S^{\prime}\right) \simeq\left\langle J_{1}, \ldots, J_{4}\right\rangle$ and we identify $H^{1}(S) \simeq\left\langle L_{1}\right\rangle \oplus\left\langle L_{2}, L_{3}, L_{4}\right\rangle$. Let $z_{i}=\tau\left(J_{i}\right) \in H^{*}\left(B S^{\prime}\right)$ and $y_{i}=\tau\left(L_{i}\right) \in H^{*}(B S)$ for $i=1, \ldots, 4$. In Section 4.11, we described the isomorphism $H^{*}\left(S^{\prime}\right) \rightarrow H^{*}(S)$ and so, under $j^{*}: H^{*}\left(B S^{\prime}\right) \rightarrow H^{*}(B S)$,

$$
j:\left\{\begin{array}{l}
z_{1} \mapsto y_{1}+y_{2}, \\
z_{2} \mapsto y_{2}-y_{1}, \\
z_{3} \mapsto y_{3}+y_{4}, \\
z_{4} \mapsto y_{3}-y_{4} .
\end{array}\right.
$$

In Section 3 we explained that $H^{*}\left(B S^{\prime}\right)^{W^{\prime}} \simeq \mathbb{Q}\left[I_{2}, I_{6}, I_{8}, I_{12}\right]$, where

$$
I_{2}=3\left(z_{1}^{2}+z_{2}^{2}+z_{3}^{2}+z_{4}^{2}\right) \text {. }
$$

Under $j^{*}: H^{*}\left(B S^{\prime}\right) \rightarrow H^{*}(B S)$,

$\frac{1}{3} I_{2} \mapsto\left(y_{1}+y_{2}\right)^{2}+\left(y_{2}-y_{1}\right)^{2}+\left(y_{3}+y_{4}\right)^{2}+\left(y_{3}-y_{4}\right)^{2}=2\left(y_{1}^{2}+y_{2}^{2}+y_{3}^{2}+y_{4}^{2}\right)$. 
By Theorem 5.1 the elements of $H^{4}(B S)^{W}$ that are in the kernel of $\alpha_{2}^{*}$ are all scalar multiples of $y_{1}^{2}+\cdots+y_{4}^{2}$. The following corollary will be used in Section 6 .

Corollary 5.21 Let $G=F_{4(4)}$. With the notation above, $\alpha_{2}^{*}\left(y_{1}^{2}\right) \neq 0$.

$\boldsymbol{F}_{4(-20)}$ Here $\operatorname{Lie}(K)=\mathfrak{s o}_{9}$ and $U=F_{4}$, and the inclusion $S \hookrightarrow S^{\prime}$ is an isomorphism. As in Section 3,

$$
H^{1}\left(S^{\prime}\right) \simeq\left\langle J_{1}, \ldots, J_{4}\right\rangle \text { and } H^{1}(S) \simeq\left\langle L_{1}, \ldots, L_{4}\right\rangle .
$$

Under $H^{1}\left(S^{\prime}\right) \rightarrow H^{1}(S)$ we have $J_{i} \mapsto L_{i}$. Let $z_{i}=\tau\left(J_{i}\right) \in H^{2}\left(B S^{\prime}\right)$ and $y_{i}=$ $\tau\left(L_{i}\right) \in H^{2}(B S)$ for $i=1, \ldots, 4$. In Section 3 we explained that $H^{*}\left(B S^{\prime}\right)^{W^{\prime}} \simeq$ $\mathbb{Q}\left[I_{2}, I_{6}, I_{8}, I_{12}\right]$, where $I_{2}=3\left(z_{1}^{2}+z_{2}^{2}+z_{3}^{2}+z_{4}^{2}\right)$. By Theorem 5.1, the elements of $H^{8}(B S)^{W}$ that are in the kernel of $\alpha_{2}^{*}$ are all scalar multiples of

$$
j^{*}\left(I_{2}\right)^{2}=9\left(\sum_{i=1}^{4} y_{i}^{4}+2 \sum_{1 \leq i<j \leq 4} y_{i}^{2} y_{j}^{2}\right) .
$$

This implies the following corollary:

Corollary 5.22 Let $G=F_{4(-20)}$. With the notation above, $\alpha_{2}^{*}\left(\sum_{1 \leq i<j \leq 4} y_{i}^{2} y_{j}^{2}\right) \neq 0$.

\subsection{Computing $\alpha_{2}^{*}$ for $G$ a real form of $G_{2}$}

$\boldsymbol{G}_{2(2)}$ Here $K=\mathrm{SU}_{2} \times \mathrm{SU}_{2}$ and $U=G_{2}$, and the inclusion $S \hookrightarrow S^{\prime}$ is an isomorphism. $H^{1}\left(S^{\prime}\right) \simeq\left\langle J_{1}, J_{2}\right\rangle$ and $H^{1}(S) \simeq\left\langle L_{1}, L_{2}\right\rangle$ and, under $H^{1}\left(S^{\prime}\right) \rightarrow H^{1}(S)$,

$$
\left\{\begin{array}{l}
J_{1} \mapsto 2 L_{1} \\
J_{2} \mapsto-3 L_{1}+L_{2}
\end{array}\right.
$$

Let $z_{i}=\tau\left(J_{i}\right)$ in $H^{2}\left(B S^{\prime}\right)$ for $i=1,2$ and $y_{i}=\tau\left(L_{i}\right)$ in $H^{2}(B S)$ for $j=1,2$.

In Section 3 we explained that $H^{*}\left(B S^{\prime}\right)^{W^{\prime}} \simeq \mathbb{Q}\left[I_{2}, I_{6}\right]$, where

$$
I_{2}=2\left(3 z_{1}^{2}+3 z_{1} z_{2}+z_{2}^{2}\right) .
$$

Under $j^{*}: H^{*}\left(B S^{\prime}\right) \rightarrow H^{*}(B S), I_{2} \mapsto 2\left(3 y_{1}^{2}+y_{2}^{2}\right)$. By Theorem 5.1, 3y $y_{1}^{2}+y_{2}^{2}$ generates the kernel of $\alpha_{2}^{*}: H^{4}\left(B G_{2(2)}\right) \rightarrow H^{4}\left(B\left(G_{2(2)}\right)^{\delta}\right)$. In particular, we have the following corollary:

Corollary 5.23 Let $G=G_{2(2)}$. With the notation above, $\alpha_{2}^{*}\left(y_{1}^{2}\right) \neq 0$. 


\section{Conclusion}

Recall that $G$ is a simple Lie group from Table 2 and $\Gamma \subset G$ is a cocompact lattice. Our goal in this section is to find nonzero elements in the image of the composition

$$
H^{*}\left(B \operatorname{Homeo}\left(S^{n-1}\right)\right) \stackrel{\alpha_{1}^{*}}{\longrightarrow} H^{*}(B G) \stackrel{\alpha_{2}^{*}}{\longrightarrow} H^{*}\left(B G^{\delta}\right) \stackrel{\alpha_{3}^{*}}{\longrightarrow} H^{*}(B \Gamma) .
$$

In Section 4 we showed that $\alpha_{1}^{*}\left(q_{i}\right)=p_{i}(\iota)=(-1)^{i} c_{2 i}(\iota \mathbb{C})$, and we computed the polynomial $c\left(\iota_{\mathbb{C}}\right)$. The kernel of $\alpha_{2}^{*}$ is generated as an ideal by the image of $i^{*}: H^{>0}\left(B G_{\mathbb{C}}\right) \rightarrow H^{>0}(B G)$ by Theorem 5.1, and $i^{*}$ was computed in Section 5. Finally, $\alpha_{3}^{*}$ is injective by the following proposition:

Proposition 6.1 Let $\Gamma \subset G$ be a cocompact lattice. Then the image of the map $H^{*}(B G) \rightarrow H^{*}\left(B G^{\delta}\right)$ injects into $H^{*}(B \Gamma)$.

The proof of Proposition 6.1 uses a transfer argument and can be found in [5, Section 2.3]. We can thus combine the computations from Sections 4 and 5 to determine if $p_{i}(\Gamma \backslash G / K) \neq 0$.

\subsection{Pontryagin classes for $\mathrm{SL}_{n}(\mathbb{R})$-manifolds}

Theorem 6.2 Let $\Gamma \subset G=\mathrm{SL}_{n}(\mathbb{R})$ be a cocompact lattice. Then $p_{i}(\Gamma \backslash G / K)=0$ for $i>0$.

Proof In Section 4.1 we computed the total Chern class $c\left(\iota_{\mathbb{C}}\right)$ of the isotropy representation. By (9) and $(10), c\left(\iota_{\mathbb{C}}\right)$ is a symmetric polynomial in $\left\{y_{1}^{2}, \ldots, y_{k}^{2}\right\}$, where $k=[n / 2]$. This implies that $c(\iota \mathbb{C})$ is in the image of

$$
H^{*}\left(B \mathrm{SL}_{n}(\mathbb{C})\right) \rightarrow H^{*}\left(B \mathrm{SL}_{n}(\mathbb{R})\right),
$$

by Section 5.1 . Then $\alpha_{2}^{*}(c(\iota \mathbb{C}))=0$ by Theorem 5.1 . Hence

$$
p_{i}(M)=\alpha_{3}^{*} \alpha_{2}^{*} \alpha_{1}^{*}\left(q_{i}\right)=(-1)^{i} \alpha_{3}^{*} \alpha_{2}^{*}\left(c\left(\iota_{\mathbb{C}}\right)\right)=0 .
$$

\subsection{Pontryagin classes for $\mathrm{SU}_{p, q}-$ manifolds}

Theorem 6.3 Let $p, q \geq 1$ and $(p, q) \neq(1,1)$. Let $G=\mathrm{SU}_{p, q}$ and let $\Gamma \subset G$ be a cocompact lattice. Then $p_{1}(\Gamma \backslash G / K) \neq 0$.

Note that $\mathrm{SU}_{1,1} \simeq \mathrm{SO}_{2,1}$. This case is covered in Theorem 6.7 . 
Proof of Theorem 6.3 From (11), one computes

$$
p_{1}(\iota)=q \sum y_{i}^{2}+p \sum z_{j}^{2}-2 \sum y_{i} z_{j}
$$

Using the relation $\sum y_{i}+\sum z_{j}=0$ in $H^{*}(B S)^{W} \simeq H^{*}(B G)$, and the relation

$$
\alpha_{2}^{*}\left(\sum y_{i}^{2}+\sum z_{j}^{2}\right)=0
$$

from Section 5.2, it follows that

$$
\alpha_{2}^{*} p_{1}(\iota)=(q-p+2) \alpha_{2}^{*}\left(\sum y_{i}\right)^{2}+2(p-q) \alpha_{2}^{*}\left(\sum y_{i} y_{j}\right)
$$

If $q=1$, then $\alpha_{2}^{*}\left(\sum y_{i}\right)^{2}=\alpha_{2}^{*}\left(\sum y_{i} y_{j}\right)$ by Corollary 5.4 and so

$$
\alpha_{2}^{*} p_{1}(\iota)=(p+1) \alpha_{2}^{*}\left(\sum y_{i} y_{j}\right)
$$

which is nonzero (again, see Corollary 5.4).

If $q \geq 2$, then $\alpha_{2}^{*}\left(\sum y_{i}\right)^{2}$ and $\alpha_{2}^{*}\left(\sum y_{i} y_{j}\right)$ are linearly independent by Corollary 5.3, and it follows that $\alpha_{2}^{*} p_{1}(\iota) \neq 0$. Hence $p_{1}(M)=\alpha_{3}^{*} \alpha_{2}^{*} p_{1}(\iota) \neq 0$ by Proposition 6.1.

\subsection{Pontryagin classes for $\operatorname{Sp}_{2 n}(\mathbb{R})-$ manifolds}

Theorem 6.4 Fix $n \geq 2$. Let $\Gamma \subset G=\operatorname{Sp}_{2 n}(\mathbb{R})$ be a cocompact lattice. Then $p_{1}(\Gamma \backslash G / K) \neq 0$.

Proof From (12), one computes

$$
p_{1}(\iota)=(n+3) \sum y_{i}^{2}+2 \sum y_{i} y_{j}
$$

By Section 5.3, $\alpha_{2}^{*}\left(\sum y_{i}^{2}\right)=0$. Using the relation $\left(\sum y_{i}\right)^{2}=\sum y_{i}^{2}+2 \sum y_{i} y_{j}$,

$$
\alpha_{2}^{*} p_{1}(\iota)=2 \alpha_{2}^{*}\left(\sum y_{i} y_{j}\right)=\alpha_{2}^{*}\left(\sum y_{i}\right)^{2}
$$

which is nonzero by Corollary 5.5. Hence $p_{1}(M)=\alpha_{3}^{*} \alpha_{2}^{*} p_{1}(\iota) \neq 0$ by Proposition 6.1.

\subsection{Pontryagin classes for $\mathrm{SO}_{p, q}-$ manifolds}

Theorem 6.5 Let $p, q \geq 2$. Let $\Gamma \subset G=\mathrm{SO}_{p, q}$ be a cocompact lattice. Then $p_{1}(\Gamma \backslash G / K) \neq 0$ if and only if $p \neq q$. 
Theorem 6.6 Fix $p \geq 4$. Let $\Gamma \subset G=\mathrm{SO}_{p, p}$ be a cocompact lattice. Then $p_{2}(\Gamma \backslash G / K) \neq 0$.

Remark $\mathrm{SO}_{2,2} \simeq \mathrm{SL}_{2}(\mathbb{R}) \times \mathrm{SL}_{2}(\mathbb{R})$ is not simple and $\mathrm{SO}_{3,3} \simeq \mathrm{SL}_{4}(\mathbb{R})$ is covered by Theorem 6.2 .

Theorem 6.7 Let $\Gamma \subset G=\mathrm{SO}_{p, 1}$ be a cocompact lattice. Then $p_{i}(\Gamma \backslash G / K)=0$ for $i>0$.

Proof of Theorem 6.5 Let $a=[p / 2]$ and $b=[q / 2]$.

Case 1 Assume $p=2 a$ and $q=2 b$ are both even. From (13) one computes

$$
p_{1}(\iota)=2 b \sum y_{i}^{2}+2 a \sum z_{j}^{2} .
$$

By Section 5.4, $\alpha_{2}^{*}\left(\sum y_{i}^{2}\right)+\alpha_{2}^{*}\left(\sum z_{j}^{2}\right)=0$, and so

$$
\alpha_{2}^{*} p_{1}(\iota)=2(b-a) \alpha_{2}^{*}\left(\sum y_{i}^{2}\right) .
$$

By Corollary 5.6, $\alpha_{2}^{*}\left(\sum y_{i}^{2}\right) \neq 0$. The assumptions that $p \neq q$ and that $p$ and $q$ are both even imply that $\alpha_{2}^{*} p_{1}(\iota) \neq 0$. Hence $p_{1}(M)=\alpha_{3}^{*} \alpha_{2}^{*} p_{1}(\iota) \neq 0$ by Proposition 6.1.

Case 2 Assume $p=2 a$ and $q=2 b+1$. From (14) one computes

$$
p_{1}(\iota)=(2 b+1) \sum y_{i}^{2}+2 a \sum z_{j}^{2} \text {. }
$$

Similar to Case 1,

$$
\alpha_{2}^{*} p_{1}(\iota)=(2 b-2 a+1) \alpha_{2}^{*}\left(\sum y_{i}^{2}\right),
$$

which is nonzero and so $p_{1}(M)=\alpha_{3}^{*} \alpha_{2}^{*} p_{1}(\iota) \neq 0$.

Case 3 Assume $p=2 a+1$ and $q=2 b$. Using (15), in an entirely similar fashion to Case 2,

$$
\alpha_{2}^{*} p_{1}(\iota)=(2 b-2 a-1) \alpha_{2}^{*}\left(\sum y_{i}^{2}\right),
$$

which is nonzero, and this implies $p_{1}(M) \neq 0$.

Case 4 Assume $p=2 a+1$ and $q=2 b+1$. From (16) one computes

$$
p_{1}(\iota)=(2 b+1) \sum y_{i}^{2}+(2 a+1) \sum z_{j}^{2} .
$$

Then

$$
\alpha_{2}^{*} p_{1}(\iota)=2(b-a) \alpha_{2}^{*}\left(\sum y_{i}^{2}\right),
$$

which is nonzero since $p \neq q$ and $p$ and $q$ are both odd. Then $p_{1}(M) \neq 0$ in this case. 
Proof of Theorem 6.6 We separate the cases when $p$ is even and when $p$ is odd.

Case 1 Assume $p=2 a$ is even. From (13) one computes

$$
\begin{aligned}
p_{2}(\iota)=\left(\begin{array}{c}
2 a \\
2
\end{array}\right) \sum y_{i}^{4}+\left(\begin{array}{c}
2 a \\
2
\end{array}\right) \sum z_{j}^{4}+(2 a)^{2} & \sum y_{i}^{2} y_{k}^{2} \\
& +(2 a)^{2} \sum z_{j}^{2} z_{\ell}^{2}+\left(4 a^{2}-6\right) \sum y_{i}^{2} z_{j}^{2} .
\end{aligned}
$$

By the computation in Section 5.4,

$$
\alpha_{2}^{*}\left(\sum y_{i}^{4}+\sum z_{j}^{4}\right)=0 \quad \text { and } \quad \alpha_{2}^{*}\left(\sum y_{i}^{2} y_{k}^{2}+\sum z_{j}^{2} z_{\ell}^{2}+\sum y_{i}^{2} z_{j}^{2}\right)=0 .
$$

From these relations, it follows that

$$
\alpha_{2}^{*} p_{2}(\iota)=-6 \alpha_{2}^{*}\left(\sum y_{i}^{2} z_{j}^{2}\right) .
$$

From the fact that $\alpha_{2}^{*}\left(\sum y_{i}^{2}+\sum z_{j}^{2}\right)^{2}=0$, it follows that

$$
\alpha_{2}^{*}\left(\sum y_{i}^{2} z_{j}^{2}\right)=\alpha_{2}^{*}\left(\sum y_{i}^{2}\right)^{2}
$$

By Corollary 5.7, $\alpha_{2}^{*}\left(\sum y_{i}^{2}\right)^{2} \neq 0$ for $p \geq 4$, so we conclude from (35) and (36) that $\alpha_{2}^{*} p_{2}(\iota) \neq 0$. Then $p_{2}(M)=\alpha_{3}^{*} \alpha_{2}^{*} p_{2}(\iota) \neq 0$ by Proposition 6.1 .

Case 2 Assume $p=2 a+1$ is odd. The total Chern class of the isotropy representation is given in (16). Let $A=\prod_{i=1}^{a}\left(1-y_{i}^{2}\right) \prod_{j=1}^{a}\left(1-z_{j}^{2}\right)$. From the computation in Section 5.4 (combined with Theorem 5.1), it is immediate that $\alpha_{2}^{*}(A)=1$. Then the computation of $\alpha_{2}^{*} p_{2}(\iota)$ is the exact same as in Case 1. Again we conclude $p_{2}(M) \neq 0$.

Proof of Theorem 6.7 If $G=\mathrm{SO}_{p, 1}$, then $b=0$ and the total Chern class is a symmetric polynomial in $\left\{y_{1}^{2}, \ldots, y_{a}^{2}\right\}$. In Section 5.4, we saw that all such polynomials are in the image of $H^{*}\left(B G_{\mathbb{C}}\right) \rightarrow H^{*}(B G)$, and hence in the kernel of $\alpha_{2}^{*}$ by Theorem 5.1. This implies $p_{i}(M)=0$ for all $i \geq 1$.

\subsection{Pontryagin classes for $\mathrm{Sp}_{p, q}$-manifolds}

It is worth noting the similarity between $\mathrm{Sp}_{p, q}$ and $\mathrm{SO}_{2 p, 2 q}$. On the level of cohomology,

while

$$
H^{*}\left(B \operatorname{Sp}_{p, q}\right) \simeq \operatorname{Sym}\left(y_{1}^{2}, \ldots, y_{p}^{2}\right) \otimes \operatorname{Sym}\left(z_{1}^{2}, \ldots, z_{q}^{2}\right),
$$

$$
H^{*}\left(B \mathrm{SO}_{2 p, 2 q}\right) \simeq\left\langle\operatorname{Sym}\left(y_{1}^{2}, \ldots, y_{p}^{2}\right), y_{1} \cdots y_{p}\right\rangle \otimes\left\langle\operatorname{Sym}\left(z_{1}^{2}, \ldots, z_{q}^{2}\right), z_{1} \cdots z_{q}\right\rangle .
$$


Note also, from Sections 4.4 and 4.5, the weights of the isotropy representation for both are $\pm y_{i} \pm z_{j}$ for $i=1, \ldots, p$ and $j=1, \ldots q$. Then in both cases the total Chern class of the isotropy representation is

$$
c\left(\iota_{\mathbb{C}}\right)=\prod\left(1+y_{i}+z_{j}\right)\left(1+y_{i}-z_{j}\right)\left(1-y_{i}+z_{j}\right)\left(1-y_{i}-z_{j}\right) .
$$

Finally, by Sections 5.4 and 5.5, for $G=\mathrm{Sp}_{p, q}$ or $G=\mathrm{SO}_{2 p, 2 q}$,

$$
\operatorname{Im}\left[j^{*}: H^{*}\left(B G_{\mathbb{C}}\right) \rightarrow H^{*}(B G)\right]=\operatorname{Sym}\left(y_{1}^{2}, \ldots, y_{p}^{2}, z_{1}^{2}, \ldots, z_{q}^{2}\right),
$$

so the relations used to compute $\alpha_{2}^{*} p_{i}(\iota)$ for $\mathrm{SO}_{2 p, 2 q}$ also compute $\alpha_{2}^{*} p_{i}(\iota)$ for $\mathrm{Sp}_{p, q}$. From these observations and from the proofs of Theorems 6.5 and 6.6, we have the following two theorems. (One should replace the use of Corollaries 5.6 and 5.7 in the proofs of Theorems 6.5 and 6.6 by Corollaries 5.8 and 5.9, respectively.)

Theorem 6.8 Let $p, q \geq 1$ such that $(p, q) \neq(1,1)$. Let $\Gamma \subset G=\operatorname{Sp}_{p, q}$ be a cocompact lattice. Then $p_{1}(\Gamma \backslash G / K) \neq 0$ if and only if $p \neq q$.

Note that $\mathrm{Sp}_{1,1} \simeq \mathrm{SO}_{4,1}$, which is treated in Theorem 6.7.

Theorem 6.9 Fix $p \geq 2$. Let $\Gamma \subset G=\mathrm{Sp}_{p, p}$ be a cocompact lattice. Then $p_{2}(\Gamma \backslash G / K) \neq 0$.

\subsection{Pontryagin classes for $\mathrm{SO}_{2 n}^{*}$-manifolds}

Theorem 6.10 Fix $n \geq 3$. Let $\Gamma \subset G=\mathrm{SO}_{2 n}^{*}$ be a cocompact lattice. Then $p_{1}(\Gamma \backslash G / K) \neq 0$.

Remark We only consider $n \geq 3$ because $\mathrm{SO}_{2}^{*} \simeq \mathbb{C}$ and $\mathrm{SO}_{4}^{*}$ is not simple.

Proof From (18), one computes

$$
p_{1}(\iota)=(n-1) \sum y_{i}^{2}+2 \sum y_{i} y_{j} .
$$

By the computation in Section 5.6, $\alpha_{2}^{*}\left(\sum y_{i}^{2}\right)=0$. Using the relation $\left(\sum y_{i}\right)^{2}=$ $\sum y_{i}^{2}+2 \sum y_{i} y_{j}$, it follows that

$$
\alpha_{2}^{*} p_{1}(\iota)=2 \alpha_{2}^{*}\left(\sum y_{i} y_{j}\right)=\alpha_{2}^{*}\left(\sum y_{i}\right)^{2},
$$

which is nonzero by Corollary 5.10. Then $p_{1}(M)=\alpha_{3}^{*} \alpha_{2}^{*} p_{1}(\iota) \neq 0$ by Proposition 6.1. 


\subsection{Pontryagin classes for $\mathrm{SU}_{2 n}^{*}-$ manifolds}

Theorem 6.11 Let $\Gamma \subset G=\mathrm{SU}_{2 n}^{*}$ be a cocompact lattice. Then $p_{i}(\Gamma \backslash G / K)=0$ for $i>0$.

Proof By Corollary 5.11, $\alpha_{2}^{*}$ is zero in positive degrees.

\subsection{Pontryagin classes for $E_{8}-$ manifolds}

Theorem 6.12 Let $\Gamma \subset G=E_{8(8)}$ be a cocompact lattice. Then $p_{1}(\Gamma \backslash G / K)=0$ and $p_{2}(\Gamma \backslash G / K) \neq 0$.

Proof From Equation (20), one computes

and

$$
p_{1}(\iota)=16\left(y_{1}^{2}+\cdots+y_{8}^{2}\right)
$$

$$
p_{2}(\iota)=126 \sum_{i=1}^{8} y_{i}^{4}+244 \sum_{1 \leq i<j \leq 8} y_{i}^{2} y_{j}^{2} .
$$

By Theorem 5.1 and the computation of Section 5.8, $\sum_{i=1}^{8} y_{i}^{2}$ and $\left(\sum_{i=1}^{8} y_{i}^{2}\right)^{2}$ generate the kernel of

$$
\alpha_{2}^{*}: H^{k}\left(B E_{8(8)}\right) \rightarrow H^{k}\left(B\left(E_{8(8)}\right)^{\delta}\right)
$$

for $k=4,8$. Then $\alpha_{2}^{*} p_{1}(\iota)=0$ and

$$
\alpha_{2}^{*} p_{2}(\iota)=4 \alpha_{2}^{*}\left(\sum y_{i}^{4}\right)+122 \underbrace{\alpha_{2}^{*}\left[\left(\sum y_{i}^{2}\right)^{2}\right]}_{=0} .
$$

By Corollary 5.12, $\alpha_{2}^{*} p_{2}(\iota)=4 \alpha_{2}^{*}\left(\sum y_{i}^{4}\right) \neq 0$, and so $p_{2}(M)=\alpha_{3}^{*} \alpha_{2}^{*} p_{2}(\iota) \neq 0$ by Proposition 6.1.

Theorem 6.13 Let $\Gamma \subset G=E_{8(-24)}$ be a cocompact lattice. Then $p_{1}(\Gamma \backslash G / K) \neq 0$.

Proof From (21), one computes

$$
p_{1}(\iota)=12\left(y_{1}^{2}+\cdots+y_{6}^{2}\right)+6 y_{7}^{2}+14 y_{8}^{2} .
$$

By Theorem 5.1 and the computation of Section 5.8, $2\left(y_{1}^{2}+\cdots+y_{6}^{2}\right)+y_{7}^{2}$ generates the kernel of

Then

$$
\alpha_{2}^{*}: H^{4}\left(B E_{8(-24)}\right) \rightarrow H^{4}\left(B\left(E_{8(-24)}\right)^{\delta}\right) \text {. }
$$

$$
\alpha_{2}^{*} p_{1}(\iota)=14 \alpha_{2}^{*}\left(y_{8}^{2}\right)
$$

is nonzero by Corollary 5.13, and so $p_{1}(M)=\alpha_{3}^{*} \alpha_{2}^{*} p_{1}(\iota) \neq 0$ by Proposition 6.1. 


\subsection{Pontryagin classes for $E_{7}-$ manifolds}

Theorem 6.14 Let $\Gamma \subset G=E_{7(7)}$ be a cocompact lattice. Then $p_{1}(\Gamma \backslash G / K)=0$ and $p_{2}(\Gamma \backslash G / K) \neq 0$.

Proof From (22), one computes

and

$$
p_{1}(\iota)=595 \sum y_{i}^{2}+1210 \sum y_{i} y_{j}
$$

$$
\begin{aligned}
p_{2}(\iota)=52360 \sum y_{i}^{4}+220660 \sum & y_{i}^{3} y_{j}+336790 \sum y_{i}^{2} y_{j}^{2} \\
& +684810 \sum y_{i}^{2} y_{j} y_{k}+1392444 \sum y_{i} y_{j} y_{k} y_{\ell} .
\end{aligned}
$$

By Theorem 5.1 and the computation of Section 5.9, for $k=4,8$, the kernel of

$$
\alpha_{2}^{*}: H^{*}(B S)^{W} \simeq H^{k}\left(B E_{7(7)}\right) \rightarrow H^{k}\left(B\left(E_{7(7)}\right)^{\delta}\right)
$$

is generated by $\sum y_{i}^{2}$ and $\left(\sum y_{i}^{2}\right)^{2}$. Since $\sum y_{i}^{2}$ and $\sum y_{i} y_{j}$ are linearly dependent in $H^{*}\left(B \mathrm{SU}_{8}\right)$, it follows that $\alpha_{2}^{*} p_{1}(\iota)=0$. Using relations among the symmetric polynomials and that $\alpha_{2}^{*}\left(\sum y_{i}^{2}\right)^{2}=0$, we find that

$$
\alpha_{2}^{*} p_{2}(\iota)=-348109 \alpha_{2}^{*}\left(\sum y_{i}^{4}\right) \text {, }
$$

which is nonzero by Corollary 5.14. Then $p_{2}(M)=\alpha_{3}^{*} \alpha_{2}^{*} p_{2}(\iota) \neq 0$ by Proposition 6.1.

Theorem 6.15 Let $\Gamma \subset G=E_{7(-5)}$ be a cocompact lattice. Then $p_{1}(\Gamma \backslash G / K) \neq 0$.

Proof From (23), one computes

$$
p_{1}(\iota)=8 \sum_{i=1}^{7} y_{i}^{2} .
$$

By Theorem 5.1 and the computation of Section 5.9, the kernel of

$$
\alpha_{2}^{*}: H^{4}\left(B E_{7(-5)}\right) \rightarrow H^{4}\left(B\left(E_{7(-5)}\right)^{\delta}\right)
$$

consists of scalar multiples of $I=y_{1}^{2}+\cdots+y_{6}^{2}+2 y_{7}^{2}$, and so

$$
\alpha_{2}^{*} p_{1}(\iota)=8 \alpha_{2}^{*}\left(I-y_{7}^{2}\right)=-8 \alpha_{2}^{*}\left(y_{7}^{2}\right),
$$

which is nonzero by Corollary 5.15. Then $p_{1}(M)=\alpha_{3}^{*} \alpha_{2}^{*} p_{1}(\iota) \neq 0$ by Proposition 6.1.

Theorem 6.16 Let $\Gamma \subset G=E_{7(-25)}$ be a cocompact lattice. Then $p_{1}(\Gamma \backslash G / K) \neq 0$. 
Proof From (24), one computes

$$
p_{1}(\iota)=6\left(y_{1}^{2}+\cdots+y_{5}^{2}\right)+2 y_{6}^{2}+3 y_{7}^{2} .
$$

From Section 5.9, the kernel of

$$
\alpha_{2}^{*}: H^{4}\left(B E_{7(-25)}\right) \rightarrow H^{4}\left(B\left(E_{7(-25)}\right)^{\delta}\right)
$$

is generated by

$$
6\left(y_{1}^{2}+y_{2}^{2}+y_{3}^{2}+y_{4}^{2}+y_{5}^{2}\right)+2 y_{6}^{2}+y_{7}^{2} \text {. }
$$

Then

$$
\alpha_{2}^{*} p_{1}(\iota)=2 \alpha_{2}^{*}\left(y_{7}^{2}\right) .
$$

By Corollary 5.16, $\alpha_{2}^{*}\left(y_{7}^{2}\right) \neq 0$, and so $p_{1}(M)=\alpha_{3}^{*} \alpha_{2}^{*} p_{1}(\iota) \neq 0$ by Proposition 6.1.

\subsection{Pontryagin classes for $E_{6}-$ manifolds}

Theorem 6.17 Let $\Gamma \subset G=E_{6(6)}$ be a cocompact lattice. Then $p_{1}(\Gamma \backslash G / K)=0$ and $p_{2}(\Gamma \backslash G / K) \neq 0$.

Proof From (25), one computes

$$
\begin{aligned}
& p_{1}(\iota)=14\left(y_{1}^{2}+y_{2}^{2}+y_{3}^{2}+y_{4}^{2}\right), \\
& p_{2}(\iota)=91 \sum y_{i}^{4}+166 \sum y_{i}^{2} y_{j}^{2} .
\end{aligned}
$$

From Section 5.10, $I=y_{1}^{2}+y_{2}^{2}+y_{3}^{2}+y_{4}^{2}$ and $I^{2}$ generate the kernel of

$$
\alpha_{2}^{*}: H^{k}\left(B E_{6(6)}\right) \rightarrow H^{k}\left(B\left(E_{6(-14)}\right)^{\delta}\right)
$$

for $k=4,8$. Then $\alpha_{2}^{*} p_{1}(\iota)=0$ and

$$
\alpha_{2}^{*} p_{2}(\iota)=8 \alpha_{2}^{*}\left(\sum y_{i}^{4}\right)+83 \underbrace{\alpha_{2}^{*}\left(\sum y_{i}^{4}+2 \sum y_{i}^{2} y_{j}^{2}\right)}_{=0} .
$$

By Corollary 5.17, $\alpha_{2}^{*}\left(\sum y_{i}^{4}\right) \neq 0$, and so $p_{2}(M)=\alpha_{3}^{*} \alpha_{2}^{*} p_{2}(\iota) \neq 0$ by Proposition 6.1 .

Theorem 6.18 Let $\Gamma \subset G=E_{6(2)}$ be a cocompact lattice. Then $p_{1}(\Gamma \backslash G / K) \neq 0$.

Proof From (26), one computes

$$
p_{1}(\iota)=10 \sum_{i=1}^{6} y_{i}^{2}+20 y_{7}^{2}+8 \sum_{1 \leq i<j \leq 6} y_{i} y_{j}=6 \sum_{i=1}^{6} y_{i}^{2}+20 y_{7}^{2} .
$$


In Section 5.10, we computed that

$$
I=\sum_{i=1}^{6} y_{i}^{2}+2 y_{7}^{2}
$$

generates (as a vector space) the kernel of

$$
\alpha_{2}^{*}: H^{4}\left(B E_{6(2)}\right) \rightarrow H^{4}\left(B\left(E_{6(2)}\right)^{\delta}\right)
$$

Then

$$
\alpha_{2}^{*} p_{1}(\iota)=\alpha_{2}^{*}\left(6 I+8 y_{7}^{2}\right)=8 \alpha_{2}^{*}\left(y_{7}^{2}\right) .
$$

By Corollary 5.18, $\alpha_{2}^{*}\left(y_{7}^{2}\right) \neq 0$. Then $p_{1}(M)=\alpha_{3}^{*} \alpha_{2}^{*} p_{1}(M) \neq 0$ by Proposition 6.1.

Theorem 6.19 Let $\Gamma \subset G=E_{6(-14)}$ be a cocompact lattice. Then $p_{1}(\Gamma \backslash G / K) \neq 0$.

Proof From (27), one computes

$$
p_{1}(\iota)=4\left(y_{1}^{2}+y_{2}^{2}+y_{3}^{2}+y_{4}^{2}+y_{5}^{2}\right)+144 y_{6}^{2} .
$$

By Theorem 5.1 and the computation of Section 5.10,

$$
I=3\left(y_{1}^{2}+\cdots+y_{5}^{2}\right)+y_{6}^{2}
$$

generates the kernel of

$$
\alpha_{2}^{*}: H^{4}\left(B E_{6(-14)}\right) \rightarrow H^{4}\left(B\left(E_{6(-14)}\right)^{\delta}\right) .
$$

Since $p_{1}(\iota) \neq c \cdot I$ for any scalar $c$, we conclude that $p_{1}(\iota)$ is not in $\operatorname{ker} \alpha_{2}^{*}$. Hence $p_{1}(M)=\alpha_{3}^{*} \alpha_{2}^{*} p_{1}(\iota) \neq 0$ by Proposition 6.1.

Theorem 6.20 Let $\Gamma \subset G=E_{6(-26)}$ be a cocompact lattice. Then $p_{i}(\Gamma \backslash G / K)=0$ for $i>0$.

Proof By Corollary 5.20, $\alpha_{2}^{*}$ is zero in positive degrees.

\subsection{Pontryagin classes for $F_{4}-$ manifolds}

Theorem 6.21 Let $\Gamma \subset G=F_{4(4)}$ be a cocompact lattice. Then $p_{1}(\Gamma \backslash G / K) \neq 0$.

Proof From (29), one computes

$$
p_{1}(\iota)=14 y_{1}^{2}+10 y_{2}^{2}+10 y_{3}^{2}+10 y_{4}^{2} .
$$


By Theorem 5.1 and the computation in Section 5.11, $\sum_{i=1}^{4} y_{i}^{2}$ generates the kernel of

Then

$$
\alpha_{2}^{*}: H^{4}\left(B F_{4(-20)}\right) \rightarrow H^{4}\left(B F_{4(-20)}^{\delta}\right) .
$$

$$
\alpha_{2}^{*} p_{1}(\iota)=4 \alpha_{2}^{*}\left(y_{1}^{2}\right)+10 \underbrace{\alpha_{2}^{*}\left(y_{1}^{2}+y_{2}^{2}+y_{3}^{2}+y_{4}^{2}\right)}_{=0} .
$$

By Corollary 5.21, $\alpha_{2}^{*} p_{1}(\iota)=4 \alpha_{2}^{*}\left(y_{1}^{2}\right) \neq 0$, so $p_{1}(M)=\alpha_{3}^{*} \alpha_{2}^{*} p_{1}(\iota) \neq 0$ by Proposition 6.1 .

Theorem 6.22 Let $\Gamma \subset G=F_{4(-20)}$ be a cocompact lattice. Then $p_{1}(\Gamma \backslash G / K)=0$ and $p_{2}(\Gamma \backslash G / K) \neq 0$.

Proof From (30), one computes

$$
p_{1}(\iota)=2\left(y_{1}^{2}+\cdots+y_{4}^{2}\right) \quad \text { and } \quad p_{2}(\iota)=\frac{7}{4} \sum y_{i}^{4}+\frac{5}{2} \sum_{1 \leq i<j \leq 4} y_{i}^{2} y_{j}^{2} .
$$

By Theorem 5.1 and the computation in Section 5.11, $\sum_{i=1}^{4} y_{i}^{2}$ and $\left(\sum_{i=1}^{4} y_{i}^{2}\right)^{2}=$ $\sum y_{i}^{4}+2 \sum y_{i}^{2} y_{j}^{2}$ generate the kernel of

$$
\alpha_{2}^{*}: H^{k}\left(B F_{4(-20)}\right) \rightarrow H^{k}\left(B F_{4(-20)}^{\delta}\right)
$$

for $k=4,8$, respectively. Then $\alpha_{2}^{*} p_{1}(\iota)=0$ and

$$
\alpha_{2}^{*} p_{2}(\iota)=\frac{7}{4} \underbrace{\alpha_{2}^{*}\left(\sum y_{i}^{4}+2 \sum y_{i}^{2} y_{j}^{2}\right)}_{=0}-\frac{2}{2} \alpha_{2}^{*}\left(\sum y_{i}^{2} y_{j}^{2}\right)=-\alpha_{2}^{*}\left(\sum y_{i}^{2} y_{j}^{2}\right) \text {. }
$$

Then $\alpha_{2}^{*} p_{2}(\iota) \neq 0$ by Corollary 5.22, so $p_{2}(M)=\alpha_{3}^{*} \alpha_{2}^{*} p_{2}(\iota) \neq 0$ by Proposition 6.1 .

\subsection{Pontryagin classes for $G_{2}-$ manifolds}

Theorem 6.23 Let $\Gamma \subset G=G_{2(2)}$ be a cocompact lattice. Then $p_{1}(\Gamma \backslash G / K) \neq 0$.

Proof From (31), one computes

$$
\alpha_{2}^{*}: H^{4}\left(B G_{2(2)}\right) \rightarrow H^{4}\left(B G_{2(2)}^{\delta}\right) .
$$

Then

$$
\alpha_{2}^{*} p_{1}(\iota)=\alpha_{2}^{*}\left(8 y_{1}^{2}+12 y_{1}^{2}+4 y_{2}^{2}\right)=8 \alpha_{2}^{*}\left(y_{1}^{2}\right)+4 \underbrace{\alpha_{2}^{*}\left(3 y_{1}^{2}+y_{2}^{2}\right)}_{=0}=8 \alpha_{2}^{*}\left(y_{1}^{2}\right) .
$$


By Corollary 5.23, $\alpha_{2}^{*}\left(y_{1}^{2}\right) \neq 0$, and so $p_{1}(M)=\alpha_{3}^{*} \alpha_{2}^{*} p_{1}(\iota) \neq 0$ by Proposition 6.1.

\section{References}

[1] J F Adams, Lectures on exceptional Lie groups, University of Chicago Press, IL (1996) MR1428422

[2] W Ballmann, M Gromov, V Schroeder, Manifolds of nonpositive curvature, Progress in Mathematics 61, Birkhäuser, Boston (1985) MR823981

[3] A Borel, Topology of Lie groups and characteristic classes, Bull. Amer. Math. Soc. 61 (1955) 397-432 MR0072426

[4] A Borel, F Hirzebruch, Characteristic classes and homogeneous spaces, I, Amer. J. Math. 80 (1958) 458-538 MR0102800

[5] M Bucher, T Gelander, The generalized Chern conjecture for manifolds that are locally a product of surfaces, Adv. Math. 228 (2011) 1503-1542 MR2824562

[6] W Fulton, J Harris, Representation theory: A first course, Graduate Texts in Mathematics 129, Springer, New York (1991) MR1153249

[7] M Golubitsky, B Rothschild, Primitive subalgebras of exceptional Lie algebras, Pacific J. Math. 39 (1971) 371-393 MR0338284

[8] S Helgason, Differential geometry, Lie groups, and symmetric spaces, Pure and Applied Mathematics 80, Academic Press, New York (1978) MR514561

[9] J E Humphreys, Reflection groups and Coxeter groups, Cambridge Studies in Advanced Mathematics 29, Cambridge Univ. Press (1990) MR1066460

[10] F Kamber, P Tondeur, Flat manifolds, Lecture Notes in Mathematics 67, Springer, Berlin (1968) MR0238342

[11] R C Kirby, L C Siebenmann, Foundational essays on topological manifolds, smoothings, and triangulations, Annals of Mathematics Studies 88, Princeton Univ. Press, NJ (1977) MR0645390

[12] J-F Lafont, R Roy, A note on the characteristic classes of non-positively curved manifolds, Expo. Math. 25 (2007) 21-35 MR2286832

[13] ML Mehta, Basic sets of invariant polynomials for finite reflection groups, Comm. Algebra 16 (1988) 1083-1098 MR926338

[14] J Milnor, On the homology of Lie groups made discrete, Comment. Math. Helv. 58 (1983) 72-85 MR699007

[15] J W Milnor, J D Stasheff, Characteristic classes, Annals of Mathematics Studies 76, Princeton Univ. Press, NJ (1974) MR0440554 
[16] G D Mostow, A structure theorem for homogeneous spaces, Geom. Dedicata 114 (2005) 87-102 MR2174096

[17] M Takeuchi, On Pontrjagin classes of compact symmetric spaces, J. Fac. Sci. Univ. Tokyo Sect. I 9 (1962) 313-328 MR0145009

[18] B Tshishiku, Cohomological obstructions to Nielsen realization, J. Topol. 8 (2015) 352-376 MR3356765

Department of Mathematics, University of Chicago

Chicago, IL 60615, USA

tshishikub@math.uchicago.edu

Received: 9 April 2014 Revised: 14 December 2014 